\title{
Coupling of multiscale lattice Boltzmann discrete-element method for reactive particle fluid flows
}

\author{
Marie-Luise Maier, ${ }^{1,2}$ Ravi A. Patel $\odot, 3,4,{ }^{3}$ Nikolaos I. Prasianakis, ${ }^{5}$ Sergey V. Churakov, ${ }^{5,6}$ \\ Hermann Nirschl, ${ }^{1}$ and Mathias J. Krause ${ }^{1,2,7}$ \\ ${ }^{1}$ Institute for Mechanical Process Engineering and Mechanics, Karlsruhe Institute of Technology (KIT), Karlsruhe, Germany \\ ${ }^{2}$ Lattice Boltzmann Research Group, KIT, Karlsruhe, Germany \\ ${ }^{3}$ Institute for Concrete Structures and Building Materials and materials testing laboratory (IMB/MPA), \\ Karlsruhe Institute of Technology (KIT), Karlsruhe, Germany \\ ${ }^{4}$ formerly at Laboratory for Waste Management, Paul Scherrer Institute, Villigen, Switzerland \\ ${ }^{5}$ Laboratory for Waste Management, Paul Scherrer Institute, Villigen, Switzerland \\ ${ }^{6}$ Institute of Geological Sciences, University of Berne, Switzerland \\ ${ }^{7}$ Institute for Applied and Numerical Mathematics, KIT, Karlsruhe, Germany
}

(Received 25 May 2020; accepted 4 March 2021; published 25 March 2021)

\begin{abstract}
Reactive particulate systems are of prime importance in varieties of practical applications in process engineering. As an example this study considers extraction of phosphorous from waste water by calcium silicate hydrate particles in the P-RoC process. For such systems modeling has a large potential to help to optimize process conditions, e.g., particle-size distributions or volume flows. The goal of this study is to present a new generic modeling framework to capture relevant aspects of reactive particle fluid flows using combined lattice Boltzmann method and discrete-element method. The model developed is Euler-Lagrange scheme which consist of three-components viz., a fluid phase, a dissolved reactive substance, and suspended particles. The fluid flow and reactive mass transport are described in a continuum framework using volume-averaged Navier-Stokes and volume-averaged advection-diffusion-reaction equations, respectively, and solved using lattice Boltzmann methods. The volume-averaging procedure ensures correctness in coupling between fluid flow, reactive mass transport, and particle motion. The developed model is validated through series of well-defined benchmarks. The benchmarks include the validation of the model with experimental data for the settling of a single particle in a cavity filled with water. The benchmark to validate the multi-scale reactive transport involves comparing the results with a resolved numerical simulation. These benchmarks also prove that the proposed model is grid convergent which has previously not been established for such coupled models. Finally, we demonstrate the applicability of our model by simulating a suspension of multiple particles in fluid with a dissolved reactive substance. Comparison of this coupled model is made with a one-way coupled simulation where the influence of particles on the fluid flow and the reactive solution transport is not considered. This elucidates the need for the two-way coupled model.
\end{abstract}

DOI: 10.1103/PhysRevE.103.033306

\section{INTRODUCTION}

Reactive particulate systems are of prime importance in varieties of practical applications in process engineering. Examples of such processes include biomass conversion in photobioreactors, chemical catalytic reactors, fluidized bed reactors, and filtering systems. As a practical example of reactive particle fluid flows, this study considers the P-RoC process, that stands for phosphorus recovery by crystallization to calcium silicate hydrate (C-S-H) [1] and targets to remove phosphate from, e.g., industrial waste water by adsorption of phosphate ions on C-S-H particles. A model is needed that allows to optimize the uptake of a target substance (e.g., phosphate ions) on the particles by setting appropriate process parameters and boundary conditions.

"ravi.patel@kit.edu
There exist several approaches to model particle fluid flows. In the direct numerical simulation (DNS) approach, individual particle trajectories and interaction forces are computed with a detailed resolution of the fluid and solid particle phase. It is often applied in cases where the shape of a particle has an effect on hydrodynamics and where such an interaction is important to be fully resolved, as well as when the particle sizes are comparable to the length scale of the system of interest. Such models require extensive computational effort due to the fine resolution required for the fluid computational grid. Therefore, for the large domains, which are of practical interest for process engineering, the application of such methods is not feasible.

To account for large computational domains with large number of particles two categories of formulation exist. First category of formulation referred to as Euler-Euler approach describes particle fluid flows using pseudo continuum description. An alternative formulation which describes particle motion more accurately is the Euler-Lagrange formulation. In 
this formulation the fluid phase is considered as continuum phase and the particle phase is represented by a collection of discrete particles. The discrete particles can be considered as point particles with no interaction between them referred to as discrete-particle model. If contact forces between particles are taken into account through a particle collision model, then this is referred to as discrete element method (DEM) [2]. In both discrete-particle models and DEM, the Euler grid is at least one order of magnitude larger than the particle size to preserve assumption of volume averaging.

Within the category of Euler-Lagrange models further distinction can be made based on the coupling of the fluid and the particle phase. For certain applications where particles are small and the suspension is dilute, the influence of the particle flow on the fluid flow might be negligible. For such a case, the so called one-way coupling would suffice. It accounts for the forces that originate from the fluid and act on the particles. In contrast, in a two-way coupled model, interactions are accounted for particle-fluid interactions on the particles as well as on the fluid. In this study we focus on use of lattice Boltzmann method (LBM) for the fluid phase of the suspension, in the case of Euler-Lagrange modeling. The LBM has turned out to be an attractive alternative, due to its ability to handle complex domains and its intrinsically parallel algorithm which can scale reasonably well [3-5]. Several algorithms have been proposed for two-way coupled particle fluid flows based on the lattice Boltzmann method (LBM) [6-8]. Most of these algorithms couple particles and LBM through momentum exchange. According to Blais et al. [5], appropriate equations that model the fluid flow of dense particle fluid flows or flows in porous media are the volume-averaged Navier-Stokes (VANS) equations as they allow to handle large porosity gradients. Höcker et al. [9] developed the model of Blais et al. [5] for Euler-Euler multi-phase flows with both spatially and temporally fluctuating particle concentrations. Most studies conducted on reactive flows previously has been focused on the static solid particles which are considered as adsorbent media in packed bed reactor [10-12] or involving moving boundaries due to dissolution-precipitation reactions [13-17]. Reactions to moving particles are rare [6]. The use of consistent equations derived from volume averaging theory for reactive particle fluid flows is the key difference between the previously proposed models and the present model. In addition, we demonstrate that the proposed model is numerical grid convergent which as previously been not shown in any proposed model which uses LBM for equations in Eulerian frame.

To conclude to our knowledge at present, there is no modeling framework for fully coupled multiscale LBM and DEM for reactive particle fluid flows. Key contribution of this study is a robust generic LBM-based Euler-Lagrange algorithm for reactive particle fluid flows which handles a two-way coupling and a demonstrated numerical convergence. In this model the flow of the Newtonian fluid and the reactive transport processes are not resolved around the disperse particles but rather treated in a continuous framework through a volume-averaging procedure. The multiscale problem originate from the fact that the discrete particle size is much smaller than the macroscale domain of interest for fluid flow [18]. The fluid flow is governed by the volume-averaged
Navier-Stokes (VANS) equations which recover the DarcyBrinkman equations in systems with particles. The reactive mass transport is modeled by the volume-averaged advectiondiffusion-reaction (VAADR) equation. The dynamics of the suspended particles are modeled by the DEM. This model provides a very accurate coupling between Euler and Lagrange models. The coupling between governing equations of the fluid, the reactive mass transport and the particles considered is shown in Fig. 1. For the particles, the interaction with the fluid flow is governed by a drag force. The effect of the particles on the fluid is realized through a change of porosity and drag force. The effect of the particles on the fluid is represented through a change of porosity and drag force. For the reactive mass transport equation, the porosity is included in the diffusion coefficient, the formulation of the reaction term depends on the specific surface area. The dispersion in the diffusive solid phase is neglected.

The simulations for particle fluid flows are realized in the DEM open-source framework Yade [19] and is used for contact detection, time step determination, periodic boundary conditions, and contact laws [20,21]. Yade is coupled with the LBM-based framework Yantra [22], an open-source framework to simulate multicomponent reactive transport at the pore as well as at the meso and the continuum scale [22,23]. We demonstrate the ability of the fully coupled multiscale LBM-DEM by comparing with experimental and numerical results. In the first simulation, the settling of a single subgrid particle in a cavity filled with water is compared to the experimental results of the literature. Grid convergence studies are performed for both the fluid and the particle velocity. In a second simulation, the approach is used to compute the change in the reactive substance concentration because of reactions to subgrid particles with fixed position in a channel. It is validated by comparing the results with a simulation of fully resolved pore-scale particles of the same specific surface area [13]. Finally, the simulation is conducted for a suspension of 1000, 2000, and 3000 particles, respectively, water and reactive substance in a segment of a channel. The change in the concentration of the reactive substance at the outlet due to reaction processes is compared with the one-way coupled simulation. The article is structured as follows. First, the governing equations for the three physical processes are introduced. It is followed by details about the numerical model and implementation aspects. Finally, a series of benchmarks are presented to offer validation of the model and a demonstration case is simulated to show the potential of the developed model.

\section{GOVERNING EQUATIONS AND MODEL ASSUMPTIONS}

The model consists of three components viz., fluid (water), reactive substance, and solid particles. The flow of water and the transport of the reactive substance is modeled within the framework of Euler mechanics. Interaction with solid particles within the control volume is considered using volume-averaging theories. This results into the volume-averaged Navier-Stokes equations (VANS) and the advection-diffusion-reaction equations (VAADR). The conditions under which the methods of volume-averaging and 


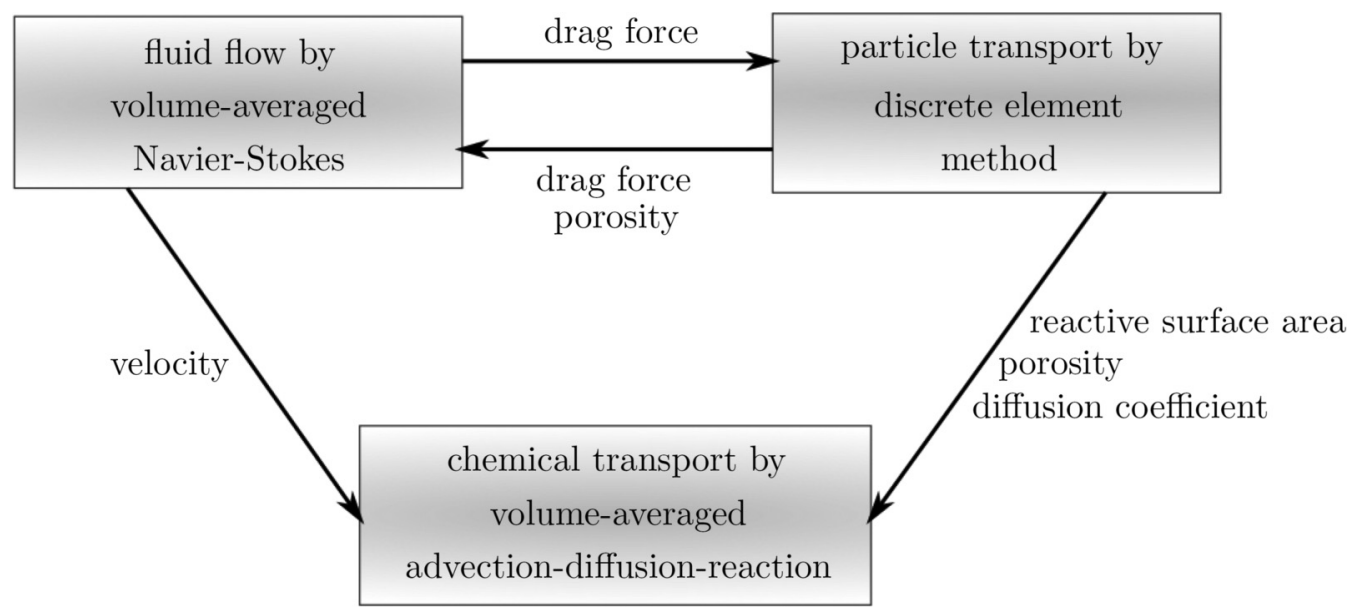

FIG. 1. Scheme of the fully coupled system of fluid, reactive substance, and particles.

averaging methods are applied are introduced by Whitaker [24]. The intrinsic volume-average of a fluid property [.] with averaging operator $\langle[\cdot]\rangle$ is defined as

$$
\langle[\cdot]\rangle=\frac{1}{V_{f}} \int_{V_{f}}[\cdot] d V,
$$

with fluid volume $V_{f} \in \mathbb{R}_{>0}\left[\mathrm{~L}^{3}\right]$ and cell volume $V \in$ $\mathbb{R}_{>0}\left[\mathrm{~L}^{3}\right][25,26]$. The dimensions are given by the corresponding symbols $L$ (length), $M$ (mass), $T$ (time), $N$ (amount of substance). The solid particles are considered spherical and their motion is modeled using the discrete element methods (DEM). The detailed formulation is presented below.

\section{A. Volume-averaged Navier-Stokes equations}

Following the volume-averaging procedure given by Whitaker [24] and Enwald et al. [25] the volume-averaged continuity equation is given as

$$
\frac{\partial \rho \phi}{\partial t}+\nabla \cdot(\rho \phi\langle\vec{u}\rangle)=0,
$$

and the corresponding volume-averaged momentum equation is given as

$$
\frac{\partial \rho \phi\langle\vec{u}\rangle}{\partial t}+(\rho \phi\langle\vec{u}\rangle \cdot \nabla)\langle\vec{u}\rangle=-\phi \nabla\langle p\rangle+\mu \nabla^{2} \phi\langle\vec{u}\rangle+\vec{f}_{\mathrm{ext}} .
$$

Equations (1) and (2) together are referred to as the volumeaveraged Navier-Stokes equations. The macroscopic quantities are the flow velocity $\vec{u}: I \times \Omega \rightarrow \mathbb{R}^{3}\left[\mathrm{~L} \mathrm{~T}^{-1}\right]$ defined on the domain $\Omega \subseteq \mathbb{R}^{3}$ with $\vec{x} \in \Omega$ and the time interval $I \subseteq \mathbb{R}$ with $t \in I[\mathrm{~T}]$, the pressure $p: I \times \Omega \rightarrow \mathbb{R}_{>0}\left[\mathrm{ML}^{-1} \mathrm{~T}^{-2}\right]$, the dynamic viscosity $\mu \in \mathbb{R}_{>0}\left[\mathrm{ML}^{-1} \mathrm{~T}^{-1}\right]$ and the fluid density $\rho \in \mathbb{R}_{>0}\left[\mathrm{ML}^{-3}\right] . \phi: I \times \Omega \rightarrow[0,1]$ is the fluid's volume fraction, also referred to as porosity of the media. A hydrodynamic drag force density $\vec{f}_{\mathrm{d}}: I \times \Omega \rightarrow$ $\mathbb{R}^{3}\left[\mathrm{ML}^{-2} \mathrm{~T}^{-2}\right]$ accounts for the back-coupling force of the solid particles to the fluid. $\vec{f}_{\text {body }}: I \times \Omega \rightarrow \mathbb{R}^{3}\left[\mathrm{ML}^{-2} \mathrm{~T}^{-2}\right]$ is an external body force density. The forces are combined in the external force density $\vec{f}_{\text {ext. }}=\vec{f}_{\mathrm{d}}+\vec{f}_{\text {body }}$.

\section{B. Volume-averaged multiscale advection-diffusion-reaction equation}

The transport of chemical substances dissolved in water can be described by an advection-diffusion-reaction equation in absence of electrokinetic effects. The reaction term in this study accounts for the adsorption reaction occurring at the surface of the particles. Applying the volume-average procedure as described in Wood et al. [27], the volume-averaged advection-diffusion-reaction equation is given as

$$
\frac{\partial \phi c}{\partial t}+\nabla \cdot\left(\phi c\langle\vec{u}\rangle-D_{\mathrm{e}} \nabla c\right)=R .
$$

The macroscopic quantity is the concentration $c: I \times \Omega \rightarrow$ $\mathbb{R}_{>0}\left[\mathrm{NL}^{-3}\right]$. In above equation the dispersion term is neglected which is a valid assumption for low to moderate Peclet numbers. The coupling with fluid flow occurs in a passivescalar manner through the averaged fluid velocity $\langle\vec{u}\rangle$ which is the solution of the VANS equation in Eqs. (1) and (2). $D_{\mathrm{e}} \in \mathbb{R}$ is the effective diffusion coefficient $\left[\mathrm{L}^{2} \mathrm{~T}^{-1}\right]$.

$D_{\mathrm{e}} \in \mathbb{R}$ is related to the molecular diffusion coefficient of reacting substances $D_{0} \in \mathbb{R}\left[\mathrm{L}^{2} \mathrm{~T}^{-1}\right]$. The relationship is derived through differential effective media theory which provides analytical expression for effective diffusion and is valid at moderate to high solid volume fractions [28,29]. It is given by $D_{\mathrm{e}}=\phi^{1.5} D_{0}$ for mono-dispersed spherical particles [30].

The term on the right-hand side of Eq. (3) is the source term $R: I \times \Omega \rightarrow \mathbb{R}\left[\mathrm{N} \mathrm{L}^{-3} \mathrm{~T}^{-1}\right]$ for reactions. For this study the adsorption process is considered as a heterogeneous reaction taking place at the fluid-solid interface. This reaction can be written as

$$
R=-k A_{s} c .
$$

Here, $A_{s}: I \times \Omega \rightarrow \mathbb{R}\left[\mathrm{L}^{-1}\right]$ is the specific surface area and $k \in \mathbb{R}\left[\mathrm{L} \mathrm{T}^{-1}\right]$ is the rate constant for a heterogeneous reaction. Additional assumptions made in deriving above rate equation considering $\mathrm{P}-\mathrm{RoC}$ process are

(1) The reaction process is kinetically controlled. The kinetics of the reaction is assumed to be linear. In the case of phosphate uptake on C-S-H particles it is a reasonable assumption as demonstrated in literature [31]. Second-order 
kinetics might provide slightly better fit for phosphate uptake in $\mathrm{C}-\mathrm{S}-\mathrm{H}$ [31]. However, as this study does not focus on developing experimentally validated simulations, a first order assumption is justifiable.

(2) Only adsorption processes are considered on the surface of the particles, desorption is neglected as there is continuous supply of phosphate in case of $\mathrm{P}-\mathrm{RoC}$ process at the inlet.

(3) The reaction, being a weak adsorption, does not change the geometry of the particle.

(4) The shape of the solid particle is spherical.

(5) The particles are mono disperse while presenting simulations in later section. However this is not the limitation of the model nor the developed framework.

\section{Discrete-element method}

The discrete-element method solves particle motion by integrating Newton's second law. Interaction between particles is modeled using contact laws, which account for forces due to particle-particle contacts and friction between particles. While coupling with the fluid flow, additional forces need to be included, e.g., hydrostatic (buoyancy force) and hydrodynamic forces (drag force), etc. Following Mordant and Pinton [32], the acting force $\vec{F}_{l}: I \rightarrow \mathbb{R}^{3}\left[\mathrm{ML} \mathrm{T}^{-2}\right]$ on a solid spherical particle in initial resting water exposed to the earth's gravity field is the sum of the drag force $\vec{F}_{d, l}$, the weight and buoyancy force $\vec{F}_{g, l}, \vec{F}_{b, l}$, the added mass force $\vec{F}_{\text {am, }, l}$, the contact force $\vec{F}_{c, l}$ and the history force. The latter is neglected here as Mordant and Pinton show that especially for the terminal stationary settling velocities the influence of history force is little. For each of the $n \in \mathbb{N}$ particles, that are assumed to be spherical and of the same size, Newton's equation of motion for force

$$
\begin{aligned}
m_{l} \frac{d \vec{u}_{l}(t)}{d t} & =\vec{F}_{l}=\vec{F}_{d, l}(t)+\vec{F}_{g, l}(t)+\vec{F}_{b, l}(t)+\vec{F}_{\mathrm{am}, l}(t)+\vec{F}_{c, l}(t), \\
\frac{d \vec{x}_{l}(t)}{d t} & =\vec{u}_{l}(t)
\end{aligned}
$$

and for torque $\vec{T}_{l}: I \rightarrow \mathbb{R}^{3}\left[\mathrm{ML}^{2} \mathrm{~T}^{-2}\right]$,

$$
J_{l} \frac{d \vec{\omega}_{l}(t)}{d t}=\vec{T}_{l}(t)
$$

is solved to obtain the trajectories and the rotational dynamics for the particles with mass $m_{l} \in \mathbb{R}_{>0}[\mathrm{M}]$, radius $r_{l} \in$ $\mathbb{R}_{>0}[\mathrm{~L}]$, particle position $\vec{x}_{l}: I \rightarrow \Omega$ [L] on the Lagrange grid, particle velocity $\vec{u}_{l}: I \rightarrow \mathbb{R}^{3}\left[\mathrm{~L} \mathrm{~T}^{-1}\right]$, moment of inertia $J_{l} \in \mathbb{R}^{3}\left[\mathrm{ML}^{2}\right]$ and the angular velocity $\vec{\omega}_{l} \in \mathbb{R}^{3}\left[\mathrm{~T}^{-1}\right], l=$ $1,2, \ldots, n$. In case of a massive sphere it is $J_{l}=2 / 5 m_{l} r_{l}^{2}$.

The drag force on particle $\vec{F}_{\mathrm{d}, l}$ results from the relative acceleration between particle and fluid,

$$
\begin{aligned}
\vec{F}_{\mathrm{d}, l}(t)= & 0.5 \rho c_{\mathrm{d}, l} \pi r_{l}^{2} \|\langle\vec{u}\rangle\left(t, \vec{x}_{l}(t)\right) \\
& -\vec{u}_{l}(t) \|\left\{\langle\vec{u}\rangle\left(t, \vec{x}_{l}(t)\right)-\vec{u}_{l}(t)\right\},
\end{aligned}
$$

with intrinsic volume averaged fluid velocity $\langle\vec{u}\rangle$ [33]. The parameter $c_{\mathrm{d}, l} \in \mathbb{R}_{>0}$ is the dimensionless drag coefficient which depends on the dimensionless particle's Reynolds num- ber $\operatorname{Re}_{l} \in \mathbb{R}_{>0}$,

$$
c_{\mathrm{d}, l}=\frac{24}{\operatorname{Re}_{1}}\left(1+0.15 \operatorname{Re}_{l}^{0.687}\right) \text { for } \operatorname{Re}_{l} \leqslant 1000 .
$$

For the formulation of the Reynolds number, the expression

$$
\operatorname{Re}_{l}=\frac{2 r_{l} \vec{u}_{\text {exp. }, l}}{0.89 \times 10^{-6} \mathrm{~m}^{2} \mathrm{~s}^{-1}}
$$

is used as given in Habte and Wu [34]. $\vec{u}_{\text {exp.,l}} \in \mathbb{R}^{3}\left[\mathrm{M} \mathrm{T}^{-1}\right.$ ] is the particular sedimentation velocity of particle $l$ obtained from experimental studies [32].

The weight and buoyancy force, the latter arises from hydrostatic pressure variation [35], is given by

$$
\vec{F}_{\mathrm{g}, l}(t)+\vec{F}_{\mathrm{b}, l}(t)=\left(m_{l}-4 / 3 \pi r_{l}^{3} \rho\right) \vec{g},
$$

with the gravitational acceleration $\vec{g} \in \mathbb{R}^{3}\left[\mathrm{~L} \mathrm{~T}^{-2}\right]$.

To account for the resistance of the surrounding water during acceleration, the added mass force

$$
\vec{F}_{\mathrm{am}, l}(t)=0.5\left(4 / 3 \pi r_{l}^{3} \rho\right)\left\{\frac{D\langle\vec{u}\rangle\left(t, \vec{x}_{l}(t)\right)}{D t}-\frac{d \vec{u}_{l}(t)}{d t}\right\}
$$

is considered as given in Refs. [32,36].

In Yade, the contact force $\vec{F}_{\mathrm{c}, l}$ is taken into account by contact laws of Cundall and Strack [2] and is given by the sum of the contact forces $\vec{F}_{c, l k}$ of the particles $k$ that are in contact with particle $l, \vec{F}_{c, l}(t)=\sum_{k=1, k \neq l}^{k_{l}} \vec{F}_{c, l k}$ with $k=$ $1,2, \ldots, k_{l} \in \mathbb{N}_{\leqslant n}$ [37]. It is split in the normal and the shear force,

$$
\begin{gathered}
\vec{F}_{c, l k}(t)=\vec{F}_{n, l k}(t)+\vec{F}_{t, l k}(t), \\
\vec{F}_{n, l k}(t)=k_{n, l k} \delta_{n, l k} \vec{n}_{l k}, \\
\vec{F}_{t, l k}(t)=k_{t, l k} \vec{\delta}_{t, l k} .
\end{gathered}
$$

Both depend on parameters of the interacting particles, normal $k_{n, l k} \in \mathbb{R}\left[\mathrm{ML}^{-2} \mathrm{~T}^{-2}\right]$ and shear stiffness $k_{t, l k} \in$ $\mathbb{R}\left[\mathrm{ML}^{-2} \mathrm{~T}^{-2}\right]$, normal $\delta_{n, l k} \in \mathbb{R}_{\geqslant 0}[\mathrm{M}]$ and shear displacement $\vec{\delta}_{t, l k} \in \mathbb{R}_{\geqslant 0}^{3}[\mathrm{M}]$. The latter is in perpendicular direction to the normal $\vec{n}_{l k}[-]$ of the conjunction vector of both particles. $k_{n, l k}$ and $k_{t, l k}$ are determined by the particle's material properties of the interacting particles. They are related to the macroscopic Young's modulus and the Poisson's ratio [37]. As the contact force acts on the virtual contact point of the particles, the torque is considered by

$$
\vec{T}_{l}(t)=-d_{l} \vec{n}_{l k} \times \vec{F}_{c, l k},
$$

with distance $d_{l} \in \mathbb{R}$ from particle's center to the contact point. Since in this study periodic boundaries are utilized, interactions between particles and walls are neglected. More information about the contact detection and the contact laws in Yade is given in Smilauer et al. [20,21].

\section{Coupling of the components}

The overall scheme for the coupling between different components is presented in Fig. 1, the general scheme has been discussed above. For the computation of the drag force and the added mass forces on particles in Eqs. (7) and (10), the average fluid velocity $\langle\vec{u}\rangle\left[t, \vec{x}_{l}(t)\right]$ at particle position 
$\vec{x}_{l}(t)=\left(x_{l, 1}, x_{l, 2}, x_{l, 3}\right)$ is obtained through linear interpolation commonly used by different authors [38-40] and is performed as follows:

$$
\langle\vec{u}\rangle\left(t, \vec{x}_{l}(t)\right)=\Sigma_{\vec{x} \in \Omega_{h}} s_{l}(t, \vec{x})\langle\vec{u}\rangle(t, \vec{x}),
$$

where the interpolation operator $s_{l}: I_{h} \times \Omega_{h} \rightarrow[0,1]$ is performed as

$$
s_{l}(t, \vec{x})= \begin{cases}\Pi_{d=1}^{3}\left(1-\frac{\left|x_{l, d}(t)-x_{d}\right|}{\Delta x}\right) & \text { if }\left|x_{l, d}(t)-x_{d}\right| \leqslant \Delta x \text { for } d \in\{1,2,3\}, \\ 0 & \text { otherwise. }\end{cases}
$$

Here, it is $\vec{x}=\left(x_{1}, x_{2}, x_{3}\right) \in \Omega_{h}$ on the uniform grid $\Omega_{h}$ with a given lattice spacing $\Delta x \in \mathbb{R}_{>0}$, and the product operator $\Pi$.

The drag force acting on the fluid is computed through the momentum exchange principle, i.e., the force acting on the fluid points is equal to that on the particles as given in Eq. (7) but acting in reverse direction. The force enters into Eq. (2) through the force density which is linearly interpolated on the fluid cell volume from the particle drag force as follows:

$$
\vec{f}_{d}(t, \vec{x})=-\frac{\Sigma_{l \in\{1, \ldots, n\}} s_{l}(t, \vec{x}) \vec{F}_{d, l}(t)}{\Delta x^{3}},
$$

with the volume $\Delta x^{3} \in \mathbb{R}_{>0}$ of a grid cell of the homogeneous Euler grid with spacing $\Delta x[26,41]$.

The porosity $\phi$ which accounts for the change in the volume of fluid due to presence of solid particles in the Euler grid volume in Eqs. (1), (2), and (3) is computed by the following equation:

$$
\phi(t, \vec{x})=1-\frac{\Sigma_{l \in\{1, \ldots, n\}} s_{l}(t, \vec{x}) 4 / 3 \pi r_{l}^{3}}{\Delta x^{3}} .
$$

The specific surface area $A_{\mathrm{s}}$ needed to get the source term $R$ in Eq. (3) is computed from the solid particle location,

$$
A_{s}(t, \vec{x})=\frac{\Sigma_{l \in\{1, \ldots, n\}} s_{l}(t, \vec{x}) 4 \pi r_{l}^{2}}{\Delta x^{3}} .
$$

\section{NUMERICAL APPROACH AND IMPLEMENTATION ASPECTS}

In the present study, the volume-averaged Navier-stokes and volume-averaged advection-diffusion-reaction equations are solved using a LBM and are coupled to the DEM. Therefore, lattice Boltzmann schemes for these equations are discussed further in the section below. The implementation was done with the LBM based framework Yantra as already mentioned above. For the DEM, the open source code Yade was used that utilizes the Verlet scheme to integrate Eq. (5) which is second-order accurate. For detailed description of implementation aspects, e.g., contact detection, contact force computation, etc., the readers are referred to the Yade manual [21]. The coupling between the solvers is done in staggered manner with global time step equal to the minimal time step of the DEM or LBM computation. After the initialization, the DEM computation is performed, followed by the VANS computation. Finally, the VAADR computation is run if needed. The overall coupling algorithm is as shown in Appendix B.

\section{A. Lattice Boltzmann method for volume-averaged Navier-Stokes equations}

The LBM equation for VANS is given as follows:

$$
\begin{aligned}
& f_{i}\left(t+\Delta t, \vec{x}+\vec{c}_{i} \Delta t\right) \\
& \quad=f_{i}(t, \vec{x})+\Omega_{i}^{\mathrm{BGK}}(t, \vec{x})+\Omega_{i}^{\mathrm{PCR}}(t, \vec{x})+\Omega_{i}^{\mathrm{F}}(t, \vec{x}) .
\end{aligned}
$$

The above equation depicts the evolution of the discrete particle density distribution function $f_{i}: I_{h} \times \Omega_{h} \rightarrow \mathbb{R}_{>0}$ in the discrete velocity space $\Omega_{c} \subseteq \mathbb{R}^{3}$ for a specific time $t \in I_{h} \subset I$ and position $\vec{x} \in \Omega_{h} \subset \Omega$ on the uniform grid $\Omega_{h}$ with a given lattice spacing $\Delta x \in \mathbb{R}_{>0}$, a discrete time step $\Delta t \in$ $\mathbb{R}_{>0}$ and the resolution $h \in \mathbb{N}$. During the collision step, $f_{i}$ is redistributed by the Bhatnagar-Gross-Krook (BGK) collision operator [42]

$$
\Omega_{i}^{\mathrm{BGK}}(t, \vec{x})=-\frac{\Delta t}{\tau}\left[f_{i}(t, \vec{x})-f_{i}^{\mathrm{eq}}(t, \vec{x})\right],
$$

among $q_{f} \in \mathbb{N}$ discrete lattice velocity directions $\vec{c}_{i} \in \mathbb{R}^{3}$, $i \in\left\{0, \ldots, q_{f}-1\right\}$, and relax towards the equilibrium distribution function

$$
f_{i}^{\mathrm{eq}}(t, \vec{x})=w_{i} \phi(t, \vec{x}) \rho\left\{1+\frac{\vec{c}_{i} \cdot\langle\vec{u}\rangle^{\mathrm{eq}}(t, \vec{x})}{c_{s}^{2}}-\frac{\langle\vec{u}\rangle^{\mathrm{eq}}(t, \vec{x}) \cdot\langle\vec{u}\rangle^{\mathrm{eq}}(t, \vec{x})}{2 c_{s}^{2}}+\frac{\left[\vec{c}_{i} \cdot\langle\vec{u}\rangle^{\mathrm{eq}}(t, \vec{x})\right]^{2}}{2 c_{s}^{4}}\right\},
$$

according to the relaxation time

$$
\tau=\frac{\mu}{\rho c_{s}^{2}}+\frac{\Delta t}{2}
$$

with equilibrium velocity

$$
\langle\vec{u}\rangle^{\mathrm{eq}}(t, \vec{x})=\frac{\sum_{i} f_{i}(t, \vec{x}) \vec{c}_{i}}{\phi(t, \vec{x}) \rho}+\frac{\Delta t \vec{f}_{d}(t, \vec{x})}{2 \phi(t, \vec{x}) \rho},
$$

speed of sound $c_{s} \in \mathbb{R}$ and the lattice weights $w_{i} \in \mathbb{R} . f_{i}^{\mathrm{eq}}$ is given by a Maxwell-Boltzmann distribution and is scaled by $\phi$ [43]. The second term in Eq. (22) is added to apply force density $\vec{f}_{\text {ext. }}$ using Guo forcing scheme [44]. The forcing (F) term is 
written as

$$
\Omega_{i}^{F}(t, \vec{x})=\Delta t w_{i}\left(1-\frac{\Delta t}{2 \tau}\right)\left(\frac{\vec{c}_{i}-\langle\vec{u}\rangle^{\mathrm{eq}}(t, \vec{x})}{c_{s}^{2}}+\frac{\vec{c}_{i} \cdot\langle\vec{u}\rangle^{\mathrm{eq}}(t, \vec{x})}{c_{s}^{4}} \vec{c}_{i}\right) \cdot \vec{f}_{\mathrm{ext}}(t, \vec{x}) .
$$

Furthermore, the first moments of the distribution are scaled by $\phi$ to achieve the macroscopic quantities

$$
\phi(t, \vec{x}) \rho=\sum_{i} f_{i}(t, \vec{x}) \quad \text { and } \quad \phi(t, \vec{x}) \rho\langle\vec{u}\rangle(t, \vec{x})=\sum_{i} f_{i}(t, \vec{x}) \vec{c}_{i} .
$$

The pressure is obtained by $p(t, \vec{x})=c_{s}^{2} \rho / \phi(t, \vec{x})$. It can be shown through the Chapman-Enskog analysis that the above lattice Boltzmann scheme would recover following equation in the macroscopic limits $[9,43]$,

$$
\begin{gathered}
\frac{\partial \rho \phi\langle\vec{u}\rangle}{\partial t}+(\rho \phi\langle\vec{u}\rangle \cdot \nabla)\langle\vec{u}\rangle \\
=-\nabla \phi\langle p\rangle+\mu \nabla^{2} \phi\langle\vec{u}\rangle+\vec{f}_{\mathrm{ext}} .
\end{gathered}
$$

Note that the above equation leads to incorrect pressure term for the case with varying spatial porosity. Therefore a pressure correction term has been introduced in Eq. (20). The pressure correction term (PCR) in this study is taken as follows:

$$
\Omega_{i}^{\mathrm{PCR}}(t, \vec{x})=w_{i} \rho\left[\phi\left(t, \vec{x}+\vec{c}_{i} \Delta t\right)-\phi(t, \vec{x})\right] .
$$

This term upon Taylor-series expansion and Chapman-Enskog analysis would add $\langle p\rangle \nabla \phi$ to the right-hand side of Eq. (25) thus correcting the pressure term. This is a more simple approach for implementation in the existing LB code than the modification of the streaming step through scaling as suggested in Höcker et al. [9].

For the first and third simulation, a three-dimensional (3D) D3Q19 stencil $\left(q_{f}=19\right)$ and for the second one, a twodimensional (2D) D2Q9 stencil $\left(q_{f}=9\right)$ is used for the flow simulation.

The corresponding values for $\vec{c}_{i}, w_{i}$, and $c_{s}$ are given in Appendix A.

\section{B. Lattice Boltzmann method for volume-averaged advection-diffusion-reaction equation}

For the VAADR, the two relaxation time (TRT) LBM is used $[14,23,45]$, which is given by

$g_{j}\left(t+\Delta t, \vec{x}+\vec{c}_{j} \Delta t\right)=g_{j}(t, \vec{x})+\Omega_{j}^{\mathrm{TRT}}(t, \vec{x})+\Omega_{j}^{\mathrm{RXN}}(t, \vec{x})$.

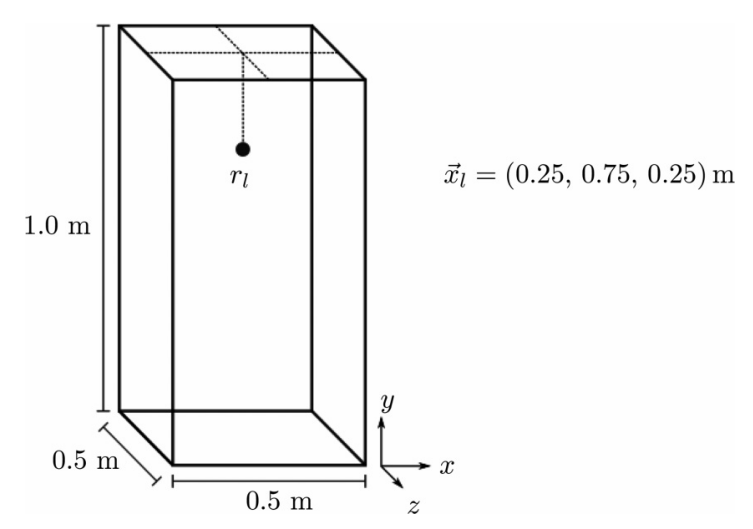

FIG. 2. Physical setup of the single-particle settling experiment.
Here, $g_{j}: I_{h} \times \Omega_{h} \rightarrow \mathbb{R}_{>0}$ is the discrete particle density distribution function for the reactive mass transport with $j \in$ $\left\{0, \ldots, q_{g}-1\right\}, q_{g} \in \mathbb{N}$ and the discrete lattice velocity directions $\vec{c}_{j} \in \mathbb{R}^{3}$. In the above equation the TRT collision operator is given by following equation

$$
\begin{aligned}
\Omega_{j}^{\mathrm{TRT}}(t, \vec{x})= & -\frac{\Delta t}{\tau^{+}}\left[g_{j}^{+}(t, \vec{x})-g_{j}^{\mathrm{eq}+}(t, \vec{x})\right] \\
& -\frac{\Delta t}{\tau^{-}}\left[g_{j}^{-}(t, \vec{x})-g_{j}^{\mathrm{eq}-}(t, \vec{x})\right],
\end{aligned}
$$

and the source or sink operator by

$$
\Omega_{j}^{\mathrm{RXN}}(t, \vec{x})=\Delta t w_{j} R(t, \vec{x}),
$$

where $w_{j}=\frac{c_{\phi}}{2}$ for $j \in\left\{1, \ldots, q_{g}-1\right\}$ and $w_{0}=\left(1-2 c_{\phi}\right)$ [45]. $c_{\phi} \in \mathbb{R}_{>0}$ is a constant parameter that is used to adjust the diffusion coefficient. It is necessary that $c_{\phi} \leqslant \frac{\min (\phi)}{3}$ [14]. Here, it is given by $c_{\phi}=\frac{1}{3.5}$.

The general idea of the TRT scheme is to relax the symmetric $\left(g_{j}^{+}\right)$, respectively, the antisymmetric $\left(g_{j}^{-}\right)$part of $g_{j}$,

$$
g_{j}^{+}=\frac{g_{j}+g_{-j}}{2}, \quad \text { respectively, } \quad g_{j}^{-}=\frac{g_{j}-g_{-j}}{2},
$$

toward the appropriate equilibrium distribution function in Eq. (27),

$$
g_{j}^{\mathrm{eq}+}=\frac{g_{j}^{\mathrm{eq}}+g_{-j}^{\mathrm{eq}}}{2}, \quad \text { respectively, } \quad g_{j}^{\mathrm{eq}-}=\frac{g_{j}^{\mathrm{eq}}-g_{-j}^{\mathrm{eq}}}{2},
$$

using the two different relaxation parameters $\tau^{+}$and $\tau^{-}$. The index $-j$ refers to the opposite velocity direction of $j \in\left\{1, \ldots, q_{g}-1\right\}$, i.e., $\vec{c}_{j}=-\vec{c}_{-j}$. The two relaxation parameters $\tau^{+}$and $\tau^{-}$are linked together through the magic parameter $(\Lambda)$ which is given as

$$
\Lambda=\left(\tau^{+}-0.5\right)\left(\tau^{-}-0.5\right)
$$

$\tau^{+}$is a free parameter which is chosen to have a stable and accurate simulation. In the present study it is set to $1 / 4$ which ensures optimal stability [46].

TABLE I. Overview over the properties of the particles that were also investigated by Mordant and Pinton [32].

\begin{tabular}{lccc}
\hline \hline Particle & $r_{l}\left[10^{-4} \mathrm{~m}\right]$ & $\rho_{l}\left[\mathrm{~kg} \mathrm{~m}^{-3}\right]$ & $\mathrm{c}_{\mathrm{d}, l}[-]$ \\
\hline Small & 2.5 & 2,560 & 1.697 \\
Medium & 7.5 & 2,560 & 0.632 \\
Large & 10.0 & 2,480 & 0.523 \\
\hline \hline
\end{tabular}


TABLE II. Comparison of the simulated terminal settling velocity $u_{l, 2}$ with the measured data $u_{\exp , l}$ of Ref. [32] and the resulting relative error.

\begin{tabular}{|c|c|c|c|c|}
\hline Particle & $u_{\mathrm{exp}, l}\left[\mathrm{~m} \mathrm{~s}^{-1}\right][32]$ & $\operatorname{Re}_{\exp , l}[-][32]$ & $u_{l, 2, h=200}(t=1 \mathrm{~s})\left[\mathrm{m} \mathrm{s}^{-1}\right]$ & $\frac{u_{l, 2, h=200}-u_{\mathrm{exp}, l}}{u_{\mathrm{exp}, l}}[-]$ \\
\hline Small & -0.074 & 41 & -0.078 & 0.0541 \\
\hline Medium & -0.218 & 360 & -0.221 & 0.0138 \\
\hline Large & -0.271 & 600 & -0.274 & 0.0111 \\
\hline
\end{tabular}

To recover Eq. (3), the appropriate equilibrium distribution function for orthogonal lattice $[23,45]$ is given as

$$
\begin{aligned}
& g_{j}^{\mathrm{eq}}(t, \vec{x})=\frac{c(t, \vec{x})}{2}\left[c_{\phi}+\phi(t, \vec{x}) \vec{c}_{j} \cdot\langle\vec{u}\rangle(t, \vec{x})\right], \\
& g_{0}^{\mathrm{eq}}(t, \vec{x})=\phi(t, \vec{x}) c(t, \vec{x})-\sum_{j>0} g_{j}^{\mathrm{eq}}(t, \vec{x}) .
\end{aligned}
$$

The zeroth moment of $g_{j}$ is

$$
\phi(t, \vec{x}) c(t, \vec{x})=\sum_{j} g_{j}(t, \vec{x}) .
$$

With above LB equation it can be shown that Eq. (3) can be recovered through the multi-scale Chapman-Enskog analysis $[23,45]$ and the effective diffusion coefficient $D_{\mathrm{e}}$ is linked to $\tau^{-}$through the following relation:

$$
D_{\mathrm{e}}=\phi(t, \vec{x}) D_{0}=c_{\phi}\left(\tau^{-}-\frac{\Delta t}{2}\right) .
$$

For the first and third simulation, a D3Q7 stencil $\left(q_{g}=7\right)$ and for the second simulation, a D2Q5 stencil $\left(q_{g}=5\right)$ is used for the reactive mass transport. The corresponding properties of the stencils are given in the Appendix.

\section{RESULTS AND DISCUSSION}

In this section, the validation of the model and the results from numerical experiments are presented. First, two way coupling between VANS and DEM is validated by comparison with experiments in literature. It is also demonstrated that the proposed model is convergent on grid refinement. This has not been demonstrated for any models proposed in literature before. Second, the validation is provided for the use of the VAADR to model the reactive transport process in presence of subgrid particles. To achieve this, a direct comparison with a fully resolved pore-scale simulation is presented. Finally, a numerical experiment is performed to illustrate the working and applicability of the fully coupled model.

\section{A. Single-particle settlement simulation for validation of VANS and DEM two-way coupling}

Two-way coupling of VANS and DEM was validated against the experiments of Mordant and Pinton [32]. They investigated the settling of a single particle in resting water $\left(\rho=1000 \mathrm{~kg} \mathrm{~m}^{-3}, \mu=0.89 \times 10^{-4} \mathrm{~kg} \mathrm{~m}^{-1} \mathrm{~s}^{-1}\right)$ by experimental studies. The size of the simulation domain is chosen to be $(0.5,1.0,0.5) \mathrm{m}$ for the $(x, y, z)$ direction, Fig. 2 . Table I gives an overview of the radius $r_{l}$ and the density $\rho_{l}$ of three exemplary particles of small, medium and large size. It also provides the corresponding drag coefficients $\mathrm{c}_{d, l}$.
Drag coefficients are computed using Eq. (8) and $u_{\text {exp., },}$, (given in Table II). The initial particle position is $\vec{x}_{l}=(0.25,0.75$, $0.25) \mathrm{m}$, the initial particle and fluid velocity is set to zero in all directions. The gravitational acceleration is taken as $\vec{g}=(0,9.80665,0) \mathrm{m} \mathrm{s}^{-2}$. The duration of the simulation is $t=1 \mathrm{~s}$. For DEM all boundaries are set as periodic. For fluid flow the boundaries in $x$ - and $z$-direction are periodic and in $y$ direction a zero flux boundary is applied through half-way bounce-back $[47,48]$. The dimensionless relaxation time is $\tau=0.50006$ which is kept constant while performing the grid convergence study. Doing so, both $\Delta x$ and $\Delta t$ are refined successively by diffusive scaling [49].

For the grid convergence study the relative error in particle velocity $\operatorname{err}_{p}$, respective in fluid velocity $\operatorname{err}_{f}$, is computed as follows:

$$
\begin{aligned}
\operatorname{err}_{p}(h) & :=\sqrt{\frac{\sum_{t \in I_{h}}\left[u_{l, 2,200}(t)-u_{l, 2, h}(t)\right]^{2}}{\sum_{t \in I_{h}}\left[u_{l, 2,200}(t)\right]^{2}}}, \\
\operatorname{err}_{f}(h) & :=\sqrt{\frac{\sum_{\vec{x} \in \Omega_{h}}\left[\left\langle u_{2,200}\right\rangle(t, \vec{x})-\left\langle u_{2, h}\right\rangle(t, \vec{x})\right]^{2}}{\sum_{\vec{x} \in \Omega_{h}}\left[\left\langle u_{2,200}\right\rangle(t, \vec{x})\right]^{2}}},
\end{aligned}
$$

with $h<200, \quad \vec{u}_{l, h}=\left(u_{l, 1, h}, u_{l, 2, h}, u_{l, 3, h}\right) \quad$ and $\quad\left\langle\vec{u}_{h}\right\rangle=$ $\left(\left\langle u_{1, h}\right\rangle,\left\langle u_{2, h}\right\rangle,\left\langle u_{3, h}\right\rangle\right)$. For $\operatorname{err}_{p}(h)$, the particle velocities $u_{l, 2,200}$ are interpolated to the points in time, $t \in I_{h}$, of the less resolved simulation of resolution $h$. For $\operatorname{err}_{f}(h)$, the fluid velocities are interpolated to the lattice positions $\vec{x} \in \Omega_{h}$ of the less resolved simulation. In case of the particle velocity, the experimental order of convergence (EOC) is determined by the slope of the error fit line of $\operatorname{err}_{p}(h)$ over $\Delta t$. The EOC of the fluid velocity is the slope of the error fit line of $\operatorname{err}_{f}(h)$ over $\Delta x . \Delta x=0.5 \mathrm{~m} / h$ changes for $h \in\{120,140,160,180$, $200\}$ with corresponding $\Delta t \in\{1.56,1.15,0.878,0.694$, $0.562\} \times 10^{-3}$ s.

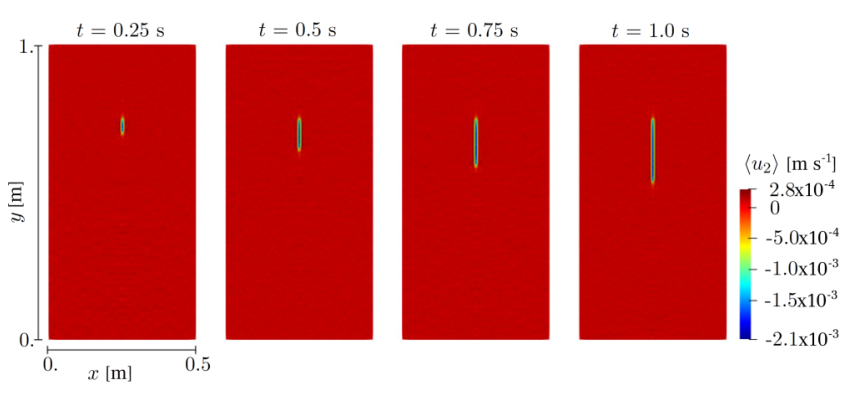

FIG. 3. The averaged velocity $\left\langle u_{2}\right\rangle$ of the fluid in $y$ direction is accelerated by the settling of the medium-sized particle. Four different times are depicted in the centered $x-y$ plane $\left(\Delta t=5.62 \times 10^{-4} \mathrm{~s}\right.$, $h=200)$. 

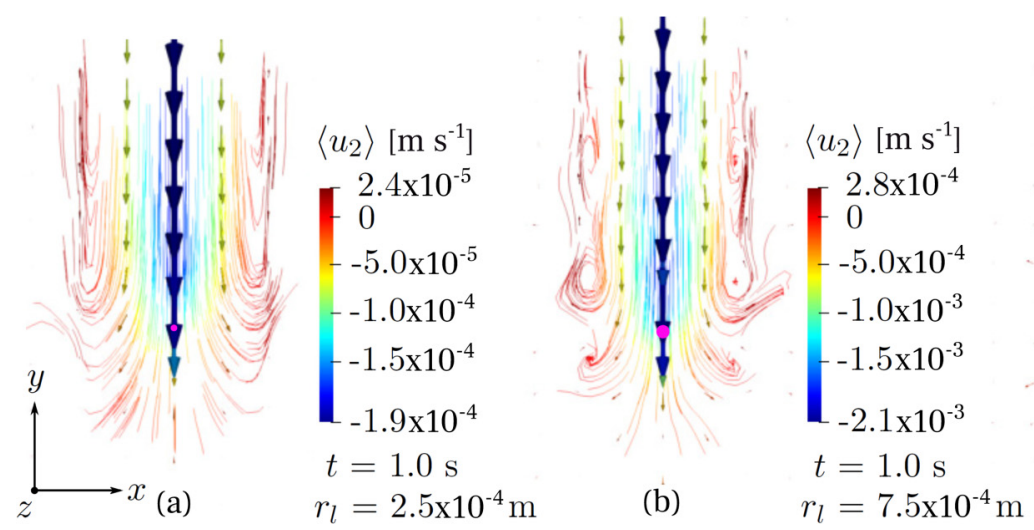

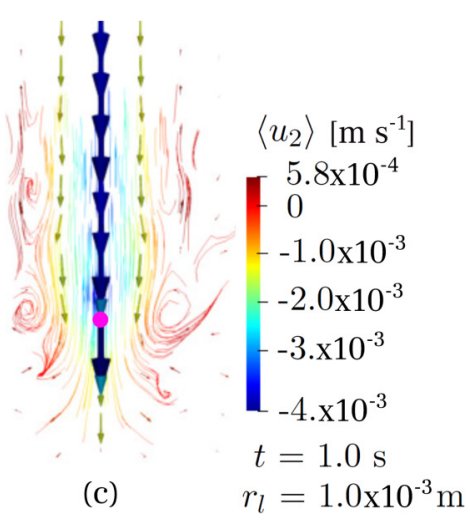

FIG. 4. Depending on the size of the violet colored particle small vortices in the fluid arise next to it $\left[\right.$ (a) $r_{l}=2.5 \times 10^{-4} \mathrm{~m}$, (b) $r_{l}=$ $\left.7.5 \times 10^{-4} \mathrm{~m},(\mathrm{c}) r_{l}=1.0 \times 10^{-3} \mathrm{~m}, \Delta t=5.62 \times 10^{-4} \mathrm{~s}, h=200\right]$. The vectors located at every Euler grid point depict the direction and are scaled by a multiple of the magnitude of the fluid velocity.

The terminal particle velocity $u_{l, 2}$ of the three particle sizes small, medium, and large $\left(h=200: \Delta t=5.62 \times 10^{-4} \mathrm{~s}\right.$, $\left.\Delta x=2.5 \times 10^{-3} \mathrm{~m}\right)$ and the measured results of Mordant and Pinton [32] show good agreement (refer to Table II). The larger the particle, the higher its negative velocity and the smaller the relative error. The latter decreases from about $5 \%$ for the small particle to about $1 \%$ for the medium and the large particle. Smaller particles reach their terminal velocity in a short time compared to larger particles. A higher temporal resolution might decrease the error. The error reported in this study is higher than that for the similar size particles carried out previously by other researchers. Maier et al. [50] reported the relative error to $u_{\text {exp. }, l}$ is about $1.8 \%$. Habte and Wu [34] enlarged the particle to $r_{l}=2.62 \times 10^{-4} \mathrm{~m}$ but compared it to $u_{\text {exp. }, l}$ of the small particle and got an error of about 3.8\%. One reason for the different results are that both studies used slightly larger $c_{d, l}=1.83$ than the one used here $\left(c_{d, l}=1.697\right)$. As $c_{\mathrm{d}, l}$ is related to $u_{\exp , l}$ by Eq. (8), this has direct impact on the results. It is remarkable that the relative error of a simulation with $c_{d, l}=1.83$ is only $1.4 \%$ as the terminal velocity is about $-0.075 \mathrm{~m} \mathrm{~s}^{-1}$. Rettinger et al. [7] run a similar simulation for a spherical particle of radius $r=$ $1.75 \times 10^{-4} \mathrm{~m}$ with density $\rho=2500 \mathrm{~kg} \mathrm{~m}^{-3}$. Their results agree very well with the experimental results of Ref. [51]. Nevertheless, nobody checked different particle sizes. Additionally, there exists no work where the EOC is computed neither for the particle nor for the fluid velocity. The effect of the two-way coupling is depicted both in the whole domain and in the direct surrounding of the particle. The settling particle induces the force density $\vec{f}_{d}$ on the fluid that is accelerated in the area touched by the particle. This is clearly visible in the velocity field plot of the fluid for the medium particle at four different times in the centered $x-y$ plane for the finest grid resolution $\left(\Delta t=5.62 \times 10^{-4} \mathrm{~s}, h=200\right)$, Fig. 3. In the case of the medium-sized particle, the trail of accelerated fluid directly behind the particle has a maximum negative velocity of $\left\langle u_{2}\right\rangle=-2.1 \times 10^{-3} \mathrm{~m} \mathrm{~s}^{-1}$. Besides the downward directed speed, the positive fluid velocity $\left\langle u_{2}\right\rangle \approx 2.8 \times 10^{-4} \mathrm{~m} \mathrm{~s}^{-1}$ occurs next to the particle, Fig. 4. For the medium and the large particle, small vortices arise next to the particle, Figs. 4(b) and 4(c). For the small particle, the transition to the back-flow is more laminar, Fig. 4(a). This behavior depends on $\operatorname{Re}_{l}$, Table II. The streamlines are computed by ParaView version 5.5.0 by use of the filter stream tracer with integrator type forward Runge-Kutta 4.

A convergence study on both the particle and the fluid velocity is performed. For the medium particle, the curves of the settling velocity over time appear to be similar for all $\Delta t$, Fig. 5. A detailed look into the transition area of acceleration state to steady state ( $t=0.09 \mathrm{~s}$ to $t=0.14 \mathrm{~s}$ ) shows that the magnitude of $u_{l, 2}$ decreases for decreasing $\Delta t$. However, for decreasing $\Delta t$ the curves approach each other. The small- and the large-sized particle show a similar behavior.

To determine whether the simulation converges, the relative error in particle velocity, $\operatorname{err}_{p}(h)$ in Eq. (32), is computed for the grid resolutions $h \in\{120,140,160,180\}$. Figure 6(a) shows the plot of error versus the time increment in a double logarithmic scaling. $\operatorname{err}_{p}(h)$ is smaller than $0.3 \%$. The slope of the errors is about 1.5 and equals the particle EOC. Theoretically, the leapfrog scheme is second order convergent. Deviation from theoretical order of convergence might stem from the forces, especially the drag force, that are time dependent and computed based on interpolation.

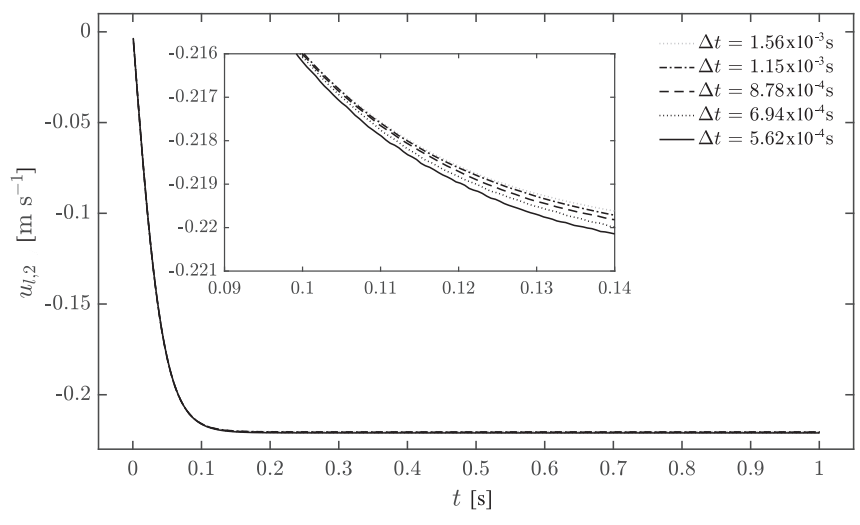

FIG. 5. For the medium-sized particle, the settling velocity over time differs little for simulations of different $\Delta t$. A convergence of the velocity towards the results of the simulation with the finest $\Delta t$ is obvious by a more detailed look. 
(a) particle error

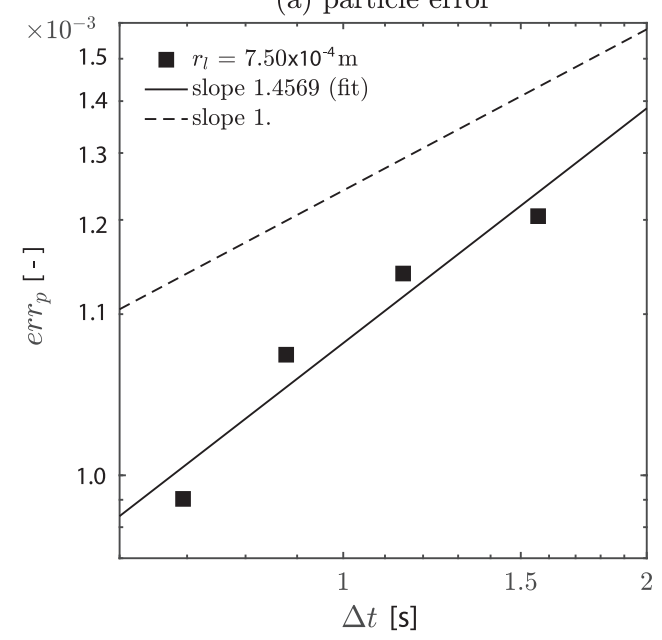

(b) fluid error

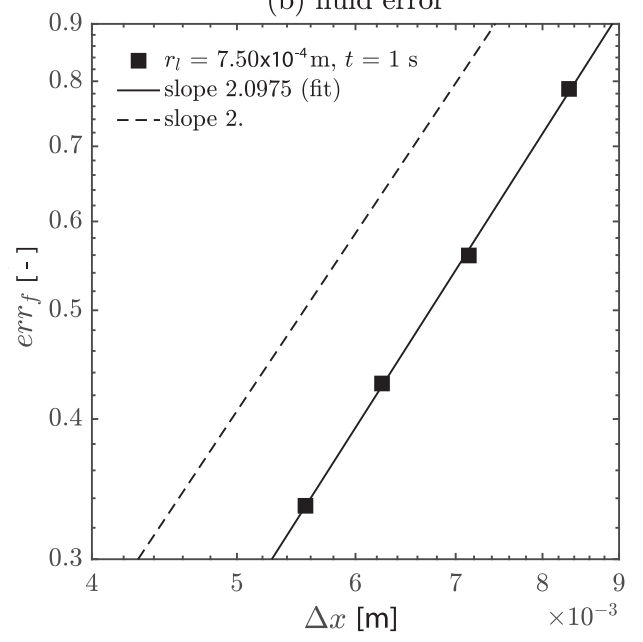

FIG. 6. (a) The relative error $\operatorname{err}_{p}(h)$ is plotted over the time increment. It is smaller than $0.3 \%$. The slope of the fit to err $p$, the particle EOC, is $\sim 1.5$. (b) The simulation converges for decreasing $\Delta x$. The slope of the fit to $\operatorname{err}_{f}$, the fluid EOC, is $\sim 2$.

For the fluid velocity grid convergence study, only grid points with velocities of larger magnitude are involved. The fast increase of the particle settling velocity creates a fast increase of the drag force acting on the fluid. That generates kind of a momentum perturbation and results in a noise on the whole grid with values of a magnitude of about $2.5 \times 10^{-5} \mathrm{~m}$ $\mathrm{s}^{-1}(h=200)$. To depict this, $\left\langle u_{2,200}\right\rangle(t=1 \mathrm{~s})$ is plotted along a horizontal line in the center of the simulation domain, $(y, z)=(0.45,0.25) \mathrm{m}$, Fig. 7 . As the noise does not directly depend on $h$, it is not considered for the error computation. The grid convergence of the fluid velocity is given by $\operatorname{err}_{f}(h)$, $h \in\{120,140,160,180\}$, Fig. 6(b). In the double logarithmic plot, the fit to the errors has a slope of $\sim 2$. The relative errors are large, however, the simulation converges. More refined simulations $(h>200)$ might be helpful to further decrease the errors while keeping $\Delta x$ larger than $r_{l}$ for the subgrid particle method $\left(h=200: \frac{\Delta x}{r_{l}}=10\right.$ for the largest particle size).

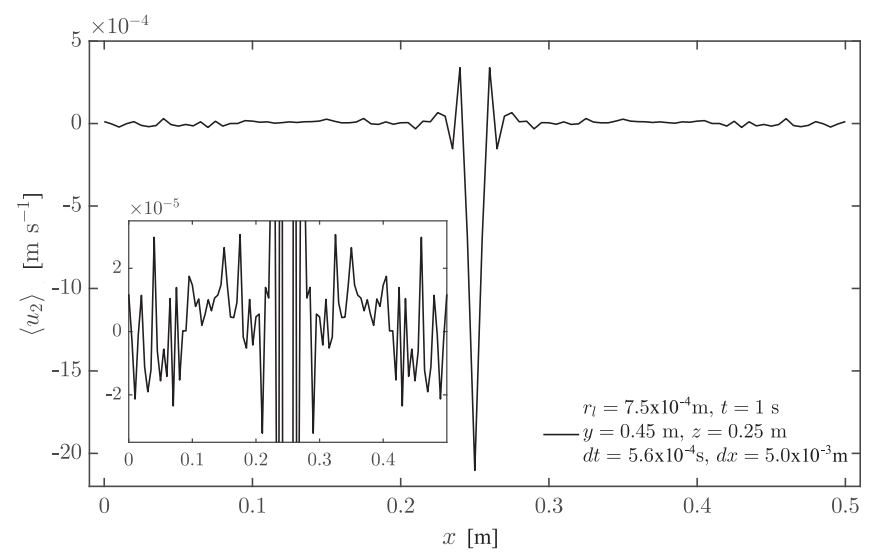

FIG. 7. The fast increase of the particle settling velocity generates kind of a momentum perturbation. This results in a noise throughout the whole domain with values of about $2.5 \times 10^{-5} \mathrm{~m} \mathrm{~s}^{-1}$ for $h=200$ for the medium-sized particle at $t=1 \mathrm{~s}$.

\section{B. Validation of an application of VANS-VAADR coupling for subgrid particles}

To validate the application of the VANS-VAADR coupling for reactive subgrid particles, a 2D test case of 50 fixed and evenly arranged particles with $r_{l}=10^{-4} \mathrm{~m}$ is chosen. The simulation from the VANS-VAADR coupled model, also referred to as unresolved simulation due to the homogenized volume representation of the particle, are compared with a fully resolved pore-scale simulations with heterogeneous reactions. For the fully resolved simulation the pore-scale modeling approach with explicit geometrical description of the particle formulated by Patel et al. [13,23] is used. There, the heterogeneous reaction is modeled as a pseudohomogeneous reaction and takes place in the fluid node adjacent to the solid node by a source term. The size of the domain is $(5.0,2.5) \times 10^{-3} \mathrm{~m}$ for the $(x, y)$-direction, Fig. 8 . The global porosity is $\phi=0.8743$. The duration of the simulation is about $2 \times 10^{-4} \mathrm{~s}$. For the test case, the properties of the fluid are set to be $\rho=1 \mathrm{~kg} \mathrm{~m}^{-3}$ and $\mu=166 \mathrm{~kg} \mathrm{~m}^{-1} \mathrm{~s}^{-1}$

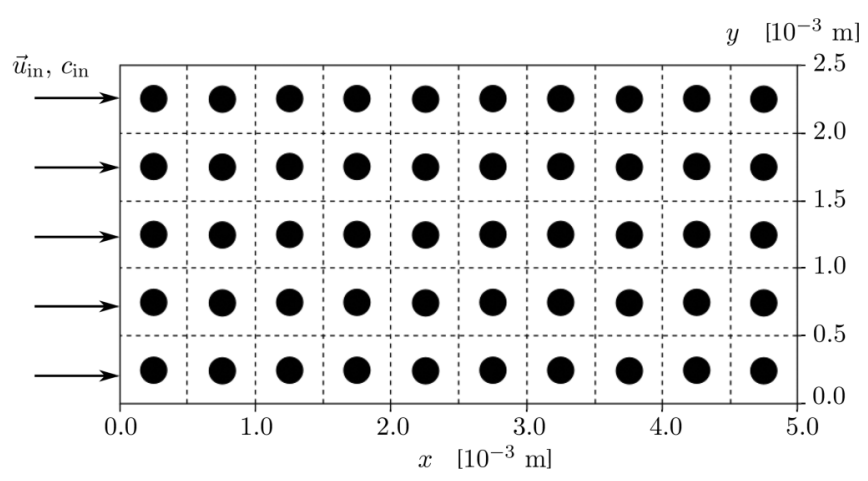

FIG. 8. The validation test case consists of a rectangular domain in $2 \mathrm{D}$ and contains 50 spherical particles where the reaction takes place. The dashed lines depict the computational grid for the coarsest resolution $\left(\Delta x=5 \times 10^{-4} \mathrm{~m}\right)$. 


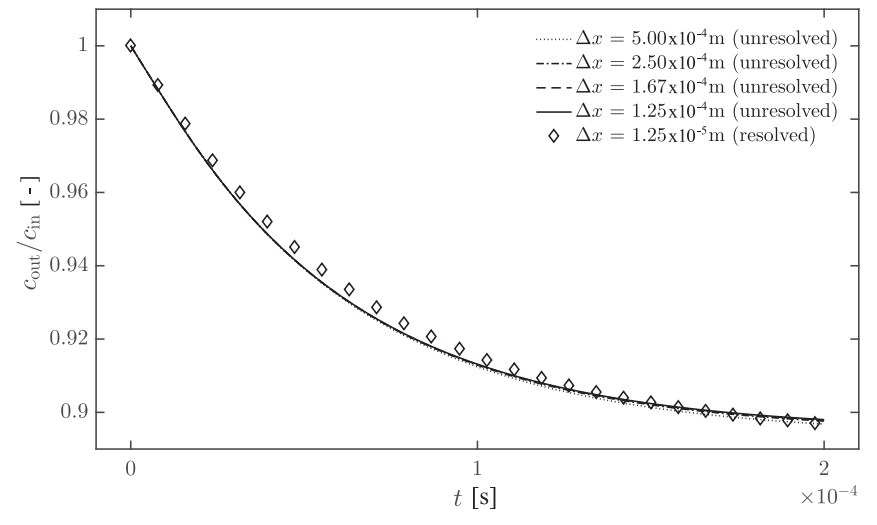

FIG. 9. Comparison of the resolved and the unresolved particle simulations for about $2 \times 10^{-4} \mathrm{~s}$.

with an initial velocity of $u_{\mathrm{in}, 2}=10^{-3} \mathrm{~m} \mathrm{~s}^{-1}$. The initial concentration of the reactive substance is $c_{\text {in }}=1 \mathrm{~mol} \mathrm{~m}^{-3}$, with $D_{0}=0.1667 \mathrm{~m}^{2} \mathrm{~s}^{-1}$ and $k=5 \times 10^{-1} \mathrm{~m} \mathrm{~s}^{-1}$. The boundary in $y$ direction is periodic for the VANS equations. For VANS, periodic fixed velocity $\left(u_{\mathrm{in}, 2}=10^{-3} \mathrm{~m} \mathrm{~s}^{-1}\right)$ at the inlet and fixed pressure at the outlet is applied in $x$ direction. For VAADR fixed concentration $\left(c_{\text {in }}=1 \mathrm{~mol} \mathrm{~m}^{-3}\right)$ at inlet and open boundary at outlet is applied. Open boundary condition for the concentration is set by copying the unknown distribution function from the next neighbor grid cell. The dimensionless relaxation time is $\tau^{-}=0.6$.

The mean concentration at the outlet normalized by the mean concentration at the inlet, $c_{\text {out }} / c_{\text {in }}$, plotted over the time decreases in an exponential manner by a tenth and reaches a steady state after about $2 \times 10^{-4} \mathrm{~s}$, Fig. 9 . Additionally, simulations are conducted for four different resolutions $\left(\Delta x=5.0 \times 10^{-4} \mathrm{~m}, 2.5 \times 10^{-4} \mathrm{~m}, 1.67 \times\right.$ $10^{-4} \mathrm{~m}, 1.25 \times 10^{-4} \mathrm{~m}$ ) for subgrid particles which are in good agreement with each other. The results of the resolved simulation $\left(\Delta x=1.25 \times 10^{-5} \mathrm{~m}\right)$ are similar but slightly larger than the unresolved values during the decrease. In transition to the steady state they are again in good agreement. The final concentration distribution in the domain is similar for both the unresolved and the resolved simulation, Fig. 10. At the inlet, $c_{\text {out }} / c_{\text {in }}=1$ and decreases to $c_{\text {out }} / c_{\text {in }}=0.885$ to 0.9 at the outlet.

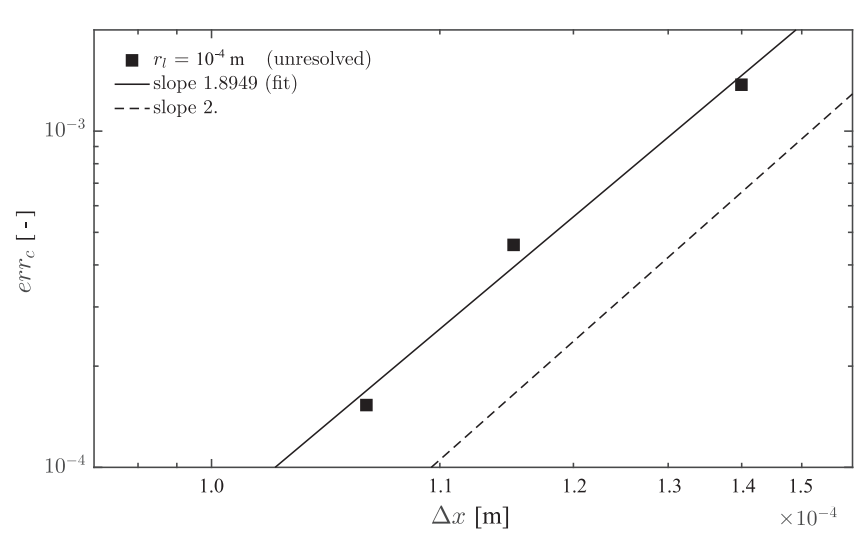

FIG. 11. The relative error $\operatorname{err}_{c}$ of the concentration at the outlet at time $t=2 \times 10^{-4} \mathrm{~s}$ is smaller than $\sim 0.1 \%$. The slope of the fit to $\operatorname{err}_{c}$ is about 2 .

To determine whether the simulation converges, the relative error in concentration, $\operatorname{err}_{c}$, is computed for the grid resolutions $h \in\{5,10,15\}$ and compared to resolution $h=$ 20. It is computed as follows:

$$
\operatorname{err}_{c}(h):=\sqrt{\frac{\sum_{\vec{x} \in \Omega_{h l} \text { lutlet }}\left[c_{20}(t, \vec{x})-c_{h}(t, \vec{x})\right]^{2}}{\sum_{\vec{x} \in \Omega_{h} l_{\text {outlet }}}\left[c_{20}(t, \vec{x})\right]^{2}}},
$$

with $h \in\{5,10,15\}$. The EOC of the concentration is the slope of the error fit line of $\operatorname{err}_{c}(h)$ over $\Delta x$. Figure 11 shows the plot of error versus $\Delta x$ in a double logarithmic scaling. $\operatorname{err}_{c}$ is smaller than $0.1 \%$. The slope of the errors, also referred to as EOC, is about two.

\section{Numerical experiment of the fully coupled model with multiple reactive particles}

This section provides demonstration of the applicability of the fully coupled VANS-VAADR-DEM model for multiparticle reactive flows. For demonstration purpose simulations are carried out on a setup of 1000, 2000, and 3000 spherical solid particles that flow in a segment of a channel of size $(5,5,5) \times 10^{-3} \mathrm{~m}$ for the $(x, y, z)$ direction, Fig. 12. The particle phase is monodisperse with radius $r_{l}=10^{-4} \mathrm{~m}$ and density $\rho_{l}=2550 \mathrm{~kg} \mathrm{~m}^{-3}$. In this setup, drag force $\vec{F}_{d, l}$ is considered to act on the particles with $c_{d, l}=1.8$. Besides,
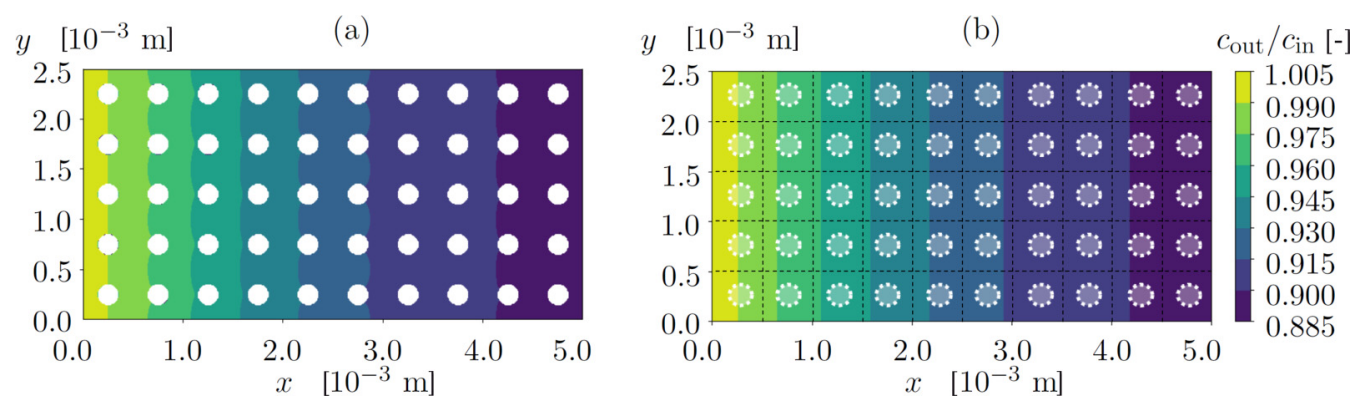

FIG. 10. Comparison of the resolved (a) and the unresolved (b) particle simulations for about $2 \times 10^{-4} \mathrm{~s}\left(\Delta x=5 \times 10^{-4} \mathrm{~m}\right)$ in a filled contour plot. In panel (b), the position of the particles is indicated by a white dotted line, the underlying grid is given by the black dotted lines. 


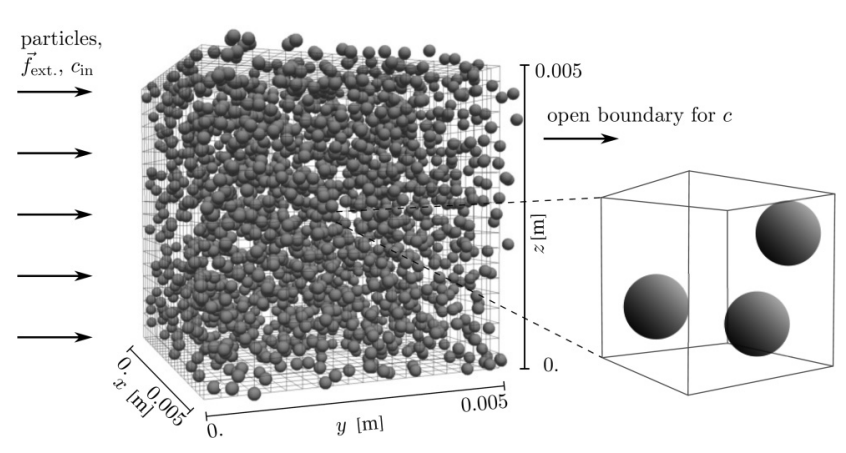

FIG. 12. Discretization of the simulation domain and depiction of 2000 particles. Some particles protrude from the box because of the periodicity of the boundaries in $x, y$, and $z$ direction for fluid and particles (left). In case of the concentration the boundaries are periodic in $y$ and $z$ direction. Crop of one Euler grid cell that contains three Lagrange particles (right).

the contact forces of particle-particle collisions are included with a Young's modulus of $10^{2} \mathrm{~kg} \mathrm{~m}^{-1} \mathrm{~s}^{-2}$ and Poisson's ratio of 0.3 . They are chosen to enable a DEM-time step size of $10^{-3} \mathrm{~s}$ and soft collisions. The properties of water are $\rho=$ $1000 \mathrm{~kg} \mathrm{~m}^{-3}$ and $\mu=10^{-4} \mathrm{~kg} \mathrm{~m}^{-1} \mathrm{~s}^{-1}$. The initial concentration of the reactive substance is $c_{\text {in }}=2.6 \times 10^{-1} \mathrm{~mol} \mathrm{~m}^{-3}$, with $D_{0}=10^{-9} \mathrm{~m}^{2} \mathrm{~s}^{-1}$ and $k=5 \times 10^{-4} \mathrm{~m} \mathrm{~s}^{-1}$. For the flow, an initial velocity $\vec{u}_{\text {in }}=\left(0,8 \times 10^{-3}, 0\right) \mathrm{m} \mathrm{s}^{-1}$ is generated by an external body force $\vec{f}_{\text {body }}=\frac{0.2}{\Delta x^{3}} \mathrm{~kg} \mathrm{~m} \mathrm{~s}^{-2}$ to mimic a pressure gradient [52]. The simulation domain consists of periodic boundaries in $x-z$ direction for the flow, the concentration and the particle simulation. For the particles and the flow, the boundaries in $y$ direction are periodic, too. The duration of the simulation is $t=2.5 \mathrm{~s}$.

First, the pure fluid velocity is computed until a steady state is reached $\left(\tau=0.7, \Delta x=2.5 \times 10^{-4} \mathrm{~m}, \Delta t=4.16 \times\right.$ $\left.10^{-3} \mathrm{~s}\right)$. In a second step, the particles are randomly distributed over the domain and are initialized with fluid velocity. Finally, the concentration is injected in the $x-z$ plane $(y=0 \mathrm{~m})\left(\tau^{-}=0.50016, \Delta x=2.5 \times 10^{-4} \mathrm{~m}, \Delta t=1.8 \times\right.$ $\left.10^{-3} \mathrm{~s}\right)$. The velocity and the concentration transport com- putation is resumed and carried on together with the particle simulation.

In Fig. 13, the concentration (a) and the magnitude of the associated velocity (b) of the 2000 particles simulation is depicted in a three dimensional manner at $t=2.4 \mathrm{~s}$. Due to the high number of particles, more particle-particle contacts occur that cause locally higher flow velocities. The two-way coupled simulation is compared to an one-way coupled system that does not consider the displacement of fluid by particles. This means the particles neither affect the fluid by a drag force nor change the diffusivity by changing porosity, i.e., $\vec{f}_{d}=0$ and $\phi=1$. The two- and one-way coupled simulations are compared for $3000(\phi=0.899), 2000$ $(\phi=0.933)$, and $1000(\phi=0.967)$ particles, Fig. 14 . In all cases, the temporal development of the mean concentration at the outlet of the domain, $c_{\text {out }}$, shows an increase after $0.5 \mathrm{~s}$. The increase is caused by the concentration transport towards the outlet. In the following seconds, $c_{\text {out }}$ fluctuates around $0.19 \mathrm{~mol} \mathrm{~m}^{-3}$ (1000 particles), $0.15 \mathrm{~mol} \mathrm{~m}^{-3}$ (2000 particles), and $0.11 \mathrm{~mol} \mathrm{~m}^{-3}$ (3000 particles). As expected, $c_{\text {out }}$ is the smallest for the 3000 particles and the largest for the 1000 particles as the reactive surface increases with increasing particle number. In case of the one-way coupling, a slight fluctuation remains for the rest of the simulation duration in all experiments. The results of the two-way coupled 1000 particles simulation are similar to the one-way coupled results. The porosity is high and thus the impact of the particles due to two way coupling is small. In case of the two-way coupled 2000 and 3000 particles simulations, the fluctuations even out and $c_{\text {out }}$ becomes larger compared to the one-way coupling results. After 1.5 to $2 \mathrm{~s}$, $c_{\text {out }}$ raises. Since the transport of chemical species to region with more particles slows down due to the two-way coupling, less reactions take place and thus $c_{\text {out }}$ increases as observed in Fig. 14 for two-way coupling case. The effect of the two-way coupled simulation is small for high porosity and increases for lower ones which is a logical outcome and qualitatively demonstrates that two way coupling is working properly.
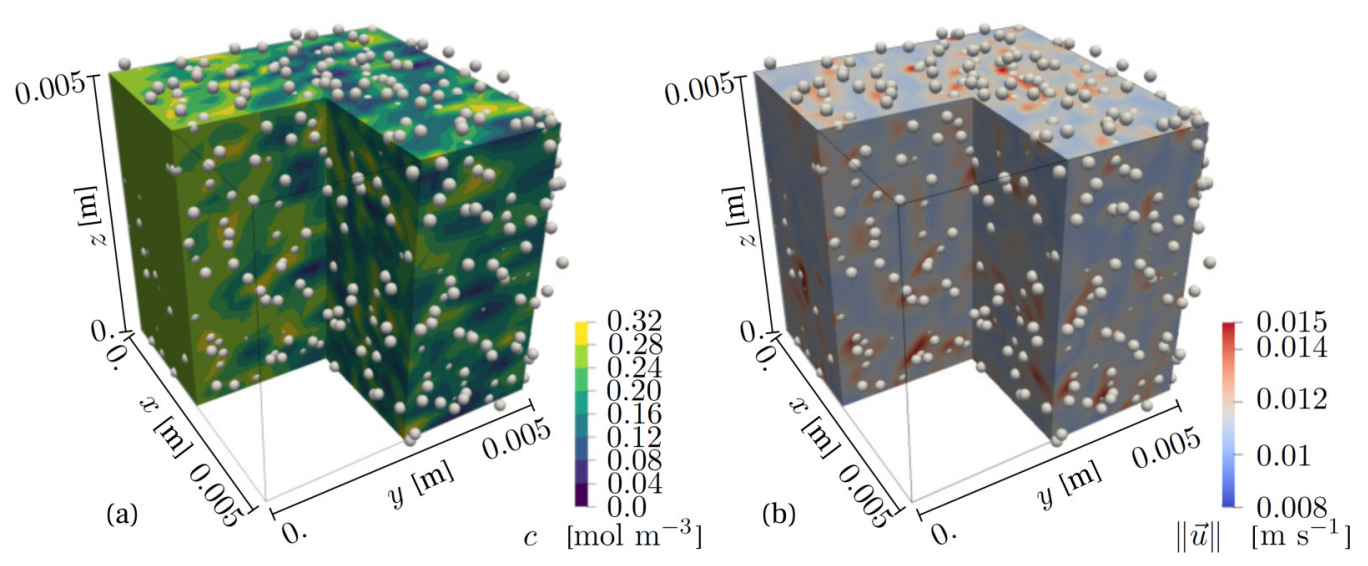

FIG. 13. Three dimensional depiction of the concentration $c$ after $t=2.4 \mathrm{~s}$ for 2000 particles (a) and magnitude of the associated velocity $\|\vec{u}\|(\mathrm{b})$. 


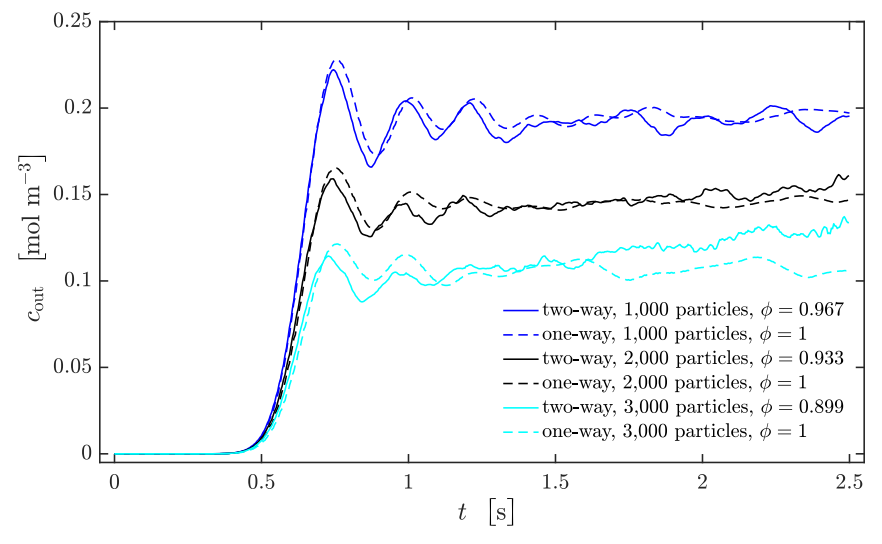

FIG. 14. Temporal progress of the mean concentration $c_{\text {out }}$ at the outlet of the channel segment. For both the one- and the two-way coupling, the concentration decreases because of the reaction that takes place at the surface of the 1000, 2000, and 3000 particles.

\section{CONCLUSION}

This work presents a fully two-way coupled multiscale lattice Boltzmann and the discrete element method of in fluid suspended multiparticles combined to reaction simulations. It recovers fluid flow and reactive transport processes around disperse and moving particles in kind of a continuum framework through a volume-averaging procedure. The main feature is the proper coupling that has been validated by experimental data from literature, comparative simulations by thoroughly tested approaches and of course grid studies. The fluid was modeled by the volumeaveraged Navier-Stokes equations, the reactive mass transport by the volume-averaged advection-diffusion-reaction equation to account for the displacement of the fluid and the reactive mass by the particles. The discrete-element method was used for the suspended, solid, subgrid particles. Fluid and particles were two-way coupled by the drag force.

The simulation of our approach show good accordance to experimental results from the literature in case of a single subgrid particle that sinks in a cavity filled with water. Performed grid studies for both the fluid and the particle velocities prove the convergence of the simulations. The simulation results of the reactive substance transport and adsorption taking place

TABLE III. In the D2Q5 lattice stencil, $c_{s}=\frac{c}{\sqrt{3}}$. In addition, there are just lattice velocities in orthogonal direction of length $c$. This lattice stencil fits for advection-diffusion problems.

\begin{tabular}{llllcc}
\hline \hline$i$ & 0 & 1 & 2 & 3 & 4 \\
\hline$w_{i}$ & $\frac{1}{3}$ & $\frac{1}{6}$ & $\frac{1}{6}$ & $\frac{1}{6}$ & $\frac{1}{6}$ \\
$c_{i, 1}$ & 0 & $c$ & 0 & $-c$ & 0 \\
$c_{i, 2}$ & 0 & 0 & $c$ & 0 & $-c$ \\
$\left\|\vec{c}_{i}\right\|$ & 0 & $c$ & $c$ & $c$ & $c$ \\
\hline \hline
\end{tabular}

TABLE IV. In the D2Q9 lattice stencil, $c_{s}=\frac{c}{\sqrt{3}}[23,53]$. In addition, the lattice velocities in orthogonal direction are of length $c$, the ones in diagonal direction are of length $\sqrt{2} c$.

\begin{tabular}{lccccccccc}
\hline \hline$i$ & 0 & 1 & 2 & 3 & 4 & 5 & 6 & 7 & 8 \\
\hline$w_{i}$ & $\frac{4}{9}$ & $\frac{1}{9}$ & $\frac{1}{9}$ & $\frac{1}{9}$ & $\frac{1}{9}$ & $\frac{1}{36}$ & $\frac{1}{36}$ & $\frac{1}{36}$ & $\frac{1}{36}$ \\
$c_{i, 1}$ & 0 & $c$ & 0 & $-c$ & 0 & $c$ & $-c$ & $-c$ & $c$ \\
$c_{i, 2}$ & 0 & 0 & $c$ & 0 & $-c$ & $c$ & $c$ & $-c$ & $-c$ \\
$\left\|\vec{c}_{i}\right\|$ & 0 & $c$ & $c$ & $c$ & $c$ & $\sqrt{2} c$ & $\sqrt{2} c$ & $\sqrt{2} c$ & $\sqrt{2} c$ \\
\hline \hline
\end{tabular}

at the surface of subgrid particles with fixed position in a channel correspond very well to the results of a fully resolved pore-scale simulation. This demonstrates the correctness of fully coupled volume-averaging process.

The multiscale approach applied to a suspension of thousands of particles demonstrates the benefit of the presented model to be an efficient simulation tool for larger particulate and reactive flow systems for industrial or engineering applications. The comparison of two-way and one-way coupling demonstrate the need for two-way coupled model to handle dense particulate systems. The encouraging results obtained in this study would pave the way to apply the model to reactive particulate systems such as the P-RoC process by numerical simulations and further validate the model with experimental studies such as the one presented in [6]. As the data is not complete (e.g., exact kinetics are not known yet) the validation is part of future work.

\section{ACKNOWLEDGMENTS}

The authors thank the Karlsruhe House of Young Scientists (KHYS) of the Karlsruhe Institute of Technology (KIT) that supported Marie-Luise Maier by the Research Travel Grants to Junior Researchers to stay at Paul Scherrer Institute (PSI), Switzerland for a research period of three months to work within the framework of her research project.

\section{APPENDIX A}

Tables III, IV, V and VI depict the associated speed of sound $c_{s}$, the weights $w_{i}$ and the velocity vectors $\vec{c}_{i}$ of the corresponding stencils with $\vec{c}_{i} \in\{-c, 0, c\}^{d}, c:=\Delta x / \Delta t$. In the two-dimensional case (2D) it is $\vec{c}_{i}=\left(c_{i, 1}, c_{i, 2}\right)$, in the three-dimensional case (3D) it is $\vec{c}_{i}=\left(c_{i, 1}, c_{i, 2}, c_{i, 3}\right)$.

TABLE V. In the D3Q7 lattice stencil, $c_{s}=\frac{c}{\sqrt{35}}$ and the lattice velocities in orthogonal direction are of length $c$ [23]. This lattice stencil fits for advection-diffusion problems.

\begin{tabular}{lccccccc}
\hline \hline$i$ & 0 & 1 & 2 & 3 & 4 & 5 & 6 \\
\hline$w_{i}$ & $\frac{1}{7}$ & $\frac{1}{7}$ & $\frac{1}{7}$ & $\frac{1}{7}$ & $\frac{1}{7}$ & $\frac{1}{7}$ & $\frac{1}{7}$ \\
$c_{i, 1}$ & 0 & $c$ & 0 & 0 & $-c$ & 0 & 0 \\
$c_{i, 2}$ & 0 & 0 & $c$ & 0 & 0 & $-c$ & 0 \\
$c_{i, 3}$ & 0 & 0 & 0 & $c$ & 0 & 0 & $-c$ \\
$\left\|\vec{c}_{i}\right\|$ & 0 & $c$ & $c$ & $c$ & $c$ & $c$ & $c$ \\
\hline \hline
\end{tabular}


TABLE VI. In the D3Q19 lattice stencil, $c_{s}=\frac{c}{\sqrt{3}}$ [53]. In addition, the lattice velocities in orthogonal direction are of length $c$, the ones in diagonal direction are of length $\sqrt{2} c$.

\begin{tabular}{lcccccccccccccccccccc}
\hline \hline$i$ & 0 & 1 & 2 & 3 & 4 & 5 & 6 & 7 & 8 & 9 & 10 & 11 & 12 & 13 & 14 & 15 & 16 & 17 & 18 \\
\hline$w_{i}$ & $\frac{1}{3}$ & $\frac{1}{18}$ & $\frac{1}{18}$ & $\frac{1}{18}$ & $\frac{1}{18}$ & $\frac{1}{18}$ & $\frac{1}{18}$ & $\frac{1}{36}$ & $\frac{1}{36}$ & $\frac{1}{36}$ & $\frac{1}{36}$ & $\frac{1}{36}$ & $\frac{1}{36}$ & $\frac{1}{36}$ & $\frac{1}{36}$ & $\frac{1}{36}$ & $\frac{1}{36}$ & $\frac{1}{36}$ & $\frac{1}{36}$ \\
$c_{i, 1}$ & 0 & $c$ & 0 & 0 & $-c$ & 0 & 0 & $-c$ & 0 & 0 & $-c$ & $-c$ & $-c$ & $c$ & $c$ & $c$ & $c$ & 0 & 0 \\
$c_{i, 2}$ & 0 & 0 & $c$ & 0 & 0 & $-c$ & 0 & 0 & $-c$ & $-c$ & $-c$ & $c$ & 0 & $c$ & $-c$ & 0 & 0 & $c$ & $c$ \\
$c_{i, 3}$ & 0 & 0 & 0 & $c$ & 0 & 0 & $-c$ & $c$ & $-c$ & $c$ & 0 & 0 & $-c$ & 0 & 0 & $c$ & $-c$ & $c$ & $-c$ \\
$\left\|\vec{c}_{i}\right\|$ & 0 & $c$ & $c$ & $c$ & $c$ & $c$ & $c$ & $\sqrt{2} c$ & $\sqrt{2} c$ & $\sqrt{2} c$ & $\sqrt{2} c$ & $\sqrt{2} c$ & $\sqrt{2} c$ & $\sqrt{2} c$ & $\sqrt{2} c$ & $\sqrt{2} c$ & $\sqrt{2} c$ & $\sqrt{2} c$ & $\sqrt{2} c$ \\
\hline \hline
\end{tabular}

\section{APPENDIX B}

Algorithm 1. Overall coupling algorithm

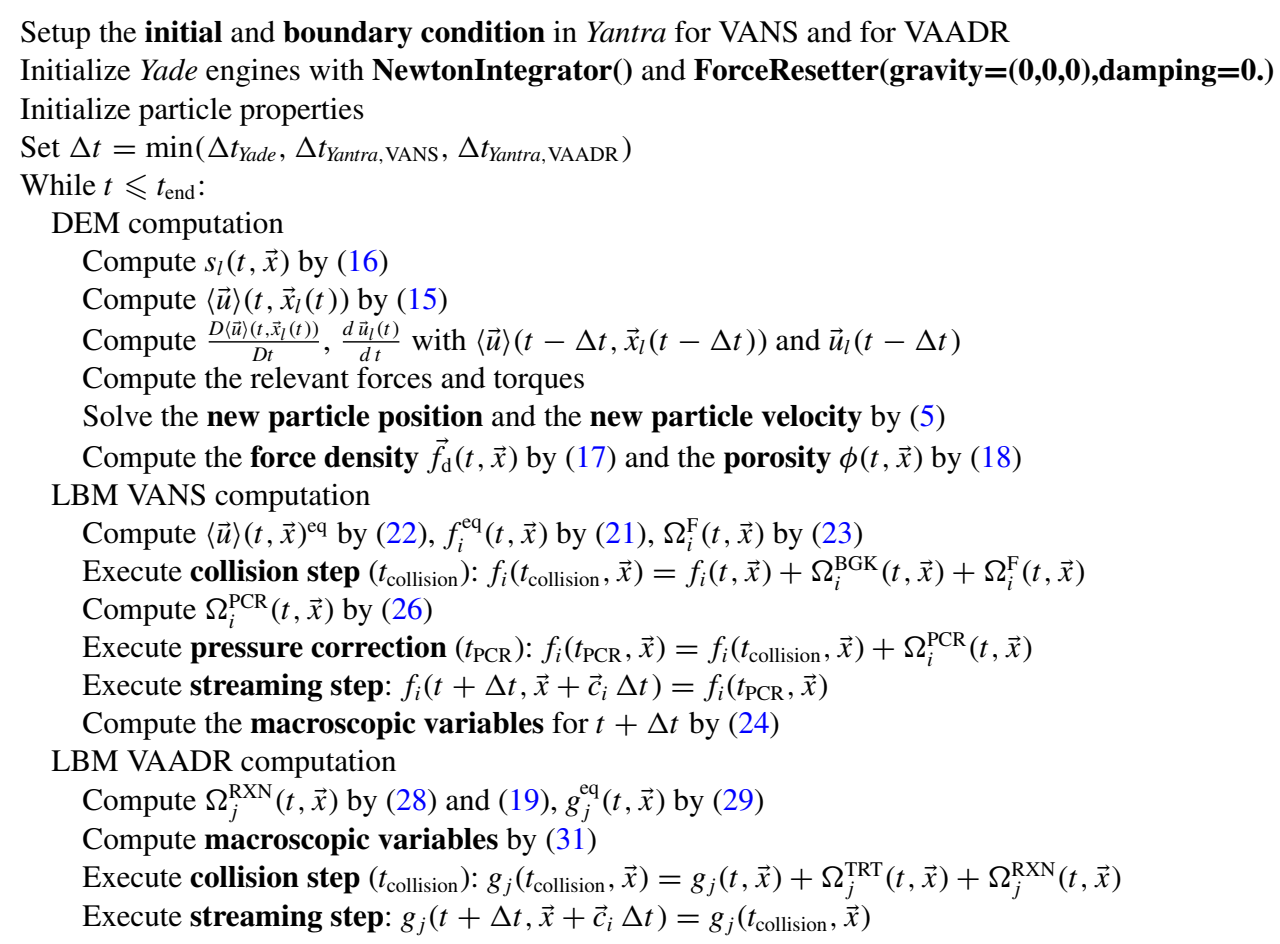

[1] U. Berg, G. Knoll, E. Kaschka, V. Kreutzer, D. Donnert, P. Weidler, and R. Nüesch, P-roc-phosphorus recovery from wastewater by crystallisation of calcium phosphate compounds, J. Residuals Sci. Technol. 4 (2005).

[2] P. A. Cundall and O. D. Strack, A discrete numerical model for granular assemblies, Geotechnique 29, 47 (1979).

[3] C. K. Aidun and J. R. Clausen, Lattice-Boltzmann method for complex flows, Annu. Rev. Fluid Mech. 42, 439 (2010).

[4] M. J. Krause, T. Gengenbach, and V. Heuveline, Hybrid parallel simulations of fluid flows in complex geometries: Application to the human lungs, in Proceedings of the European Conference on Parallel Processing (Springer, Berlin, 2010), pp. 209-216.

[5] B. Blais, J.-M. Tucny, D. Vidal, and F. Bertrand, A conservative lattice Boltzmann model for the volume-averaged
Navier-Stokes equations based on a novel collision operator, J. Comput. Phys. 294, 258 (2015).

[6] M.-L. Maier, S. Milles, S. Schuhmann, G. Guthausen, H. Nirschl, and M. J. Krause, Fluid flow simulations verified by measurements to investigate adsorption processes in a static mixer, Comput. Math. Appl. 76, 2744 (2018).

[7] C. Rettinger and U. Rüde, A coupled lattice Boltzmann method and discrete element method for discrete particle simulations of particulate flows, Comput. Fluids 172, 706 (2018).

[8] P. Ahlrichs and B. Dünweg, Simulation of a single polymer chain in solution by combining lattice Boltzmann and molecular dynamics, J. Chem. Phys. 111, 8225 (1999).

[9] S. B. Höcker, R. Trunk, W. Dörfler, and M. J. Krause, Towards the simulations of inertial dense particulate flows with 
a volume-averaged lattice Boltzmann method, Comput. Fluids 166, 152 (2018).

[10] H. Freund, T. Zeiser, F. Huber, E. Klemm, G. Brenner, F. Durst, and G. Emig, Numerical simulations of single phase reacting flows in randomly packed fixed-bed reactors and experimental validation, Chem. Eng. Sci. 58, 903 (2003).

[11] L. Zhou, Z. G. Qu, T. Ding, and J. Y. Miao, Lattice Boltzmann simulation of the gas-solid adsorption process in reconstructed random porous media, Phys. Rev. E 93, 043101 (2016).

[12] S. Sullivan, F. Sani, M. Johns, and L. Gladden, Simulation of packed bed reactors using lattice Boltzmann methods, Chem. Eng. Sci. 60, 3405 (2005).

[13] R. A. Patel, J. Perko, D. Jacques, G. De Schutter, K. Van Breugel, and G. Ye, A versatile pore-scale multicomponent reactive transport approach based on lattice Boltzmann method: Application to portlandite dissolution, Phys. Chem. Earth, Parts A/B/C 70, 127 (2014).

[14] R. A. Patel, J. Perko, D. Jacques, G. De Schutter, G. Ye, and K. Van Breugel, A three-dimensional lattice Boltzmann method based reactive transport model to simulate changes in cement paste microstructure due to calcium leaching, Constr. Build. Mater. 166, 158 (2018).

[15] R. A. Patel, S. V. Churakov, and N. I. Prasianakis, A multilevel pore scale reactive transport model for the investigation of combined leaching and carbonation of cement paste, Cem. Concr. Compos. 115, 103831 (2021).

[16] S. Molins, C. Soulaine, N. I. Prasianakis, A. Abbasi, P. Poncet, A. J. Ladd, V. Starchenko, S. Roman, D. Trebotich, H. A. Tchelepi et al., Simulation of mineral dissolution at the pore scale with evolving fluid-solid interfaces: Review of approaches and benchmark problem set, Comput. Geosci. 1 (2020).

[17] F. Gray, B. Anabaraonye, S. Shah, E. Boek, and J. Crawshaw, Chemical mechanisms of dissolution of calcite by $\mathrm{HCl}$ in porous media: Simulations and experiment, Adv. Water Resour. 121, 369 (2018).

[18] Y. Tsuji, Multi-scale modeling of dense phase gas-particle flow, Chem. Eng. Sci. 62, 3410 (2007).

[19] Yade, https://gitlab.com/yade-dev.

[20] V. Šmilauer and B. Chareyre, Yade Documentation 2nd ed, Dem formulation (The Yade Project, 2015).

[21] V. Smilauer, E. Catalano, B. Chareyre, S. Dorofeenko, J. Duriez, N. Dyck, J. Elias, B. Er, A. Eulitz, A. Gladky, C. Jakob, F. Kneib, J. Kozicki, D. Marzougui, R. Maurin, C. Modenese, L. Scholtes, L. Sibille, J. Stransky, T. Sweijen, K. Thoeni, and C. Yuan, Yade Documentation 2nd ed, Reference Manual (The Yade Project, 2015).

[22] Yantra, https://bitbucket.org/yantralbm/yantra.

[23] R. A. Patel, Lattice Boltzmann method based framework for simulating physico-chemical processes in heterogeneous porous media and its application to cement paste, $\mathrm{Ph} . \mathrm{D}$. thesis, Ghent University, 2016.

[24] S. Whitaker, The Method of Volume Averaging, Vol. 13 (Springer Science \& Business Media, Berlin, 2013).

[25] H. Enwald, E. Peirano, and A.-E. Almstedt, Eulerian two-phase flow theory applied to fluidization, Int. J. Multiphase Flow 22, 21 (1996).

[26] L. Wang, L.-P. Wang, Z. Guo, and J. Mi, Volume-averaged macroscopic equation for fluid flow in moving porous media, Int. J. Heat Mass Transf. 82, 357 (2015).

[27] B. D. Wood, F. Cherblanc, M. Quintard, and S. Whitaker, Vol- ume averaging for determining the effective dispersion tensor: closure using periodic unit cells and comparison with ensemble averaging, Water Resour. Res. 39, 1210 (2003).

[28] S. Torquato and H. Haslach Jr, Random heterogeneous materials: Microstructure and macroscopic properties, Appl. Mech. Rev. 55, B62 (2002).

[29] L. Weber, J. Dorn, and A. Mortensen, On the electrical conductivity of metal matrix composites containing high volume fractions of nonconducting inclusions, Acta Mater. 51, 3199 (2003).

[30] P. Pivonka, C. Hellmich, and D. Smith, Microscopic effects on chloride diffusivity of cement pastes-A scale-transition analysis, Cem. Concr. Res. 34, 2251 (2004).

[31] Z. Zhang, X. Wang, and J. Zhao, Phosphate recovery from wastewater using calcium silicate hydrate: Sonochemical synthesis and properties, Environ. Sci.: Water Res. Technol. 5, 131 (2019).

[32] N. Mordant and J.-F. Pinton, Velocity measurement of a settling sphere, Eur. Phys. J. B 18, 343 (2000).

[33] Y. Zhang and J. M. Reese, The drag force in two-fluid models of gas-solid flows, Chem. Eng. Sci. 58, 1641 (2003).

[34] M. A. Habte and C. Wu, Particle sedimentation using hybrid lattice Boltzmann-immersed boundary method scheme, Powder Technol. 315, 486 (2017).

[35] J. S. Marshall and S. Li, Adhesive Particle Flow (Cambridge University Press, Cambridge, UK, 2014).

[36] T. Auton, J. Hunt, and M. Prud'Homme, The force exerted on a body in inviscid unsteady nonuniform rotational flow, J. Fluid Mech. 197, 241 (1988).

[37] V. Šmilauer and B. Chareyre, Yade Documentation 2nd ed, DEM formulation (The Yade Project, 2010).

[38] S. McNamara, E. G. Flekkøy, and K. J. Måløy, Grains and gas flow: Molecular dynamics with hydrodynamic interactions, Phys. Rev. E 61, 4054 (2000).

[39] L. Goren, E. Aharonov, D. Sparks, and R. Toussaint, The mechanical coupling of fluid-filled granular material under shear, Pure Appl. Geophys. 168, 2289 (2011).

[40] A. Damsgaard, D. L. Egholm, J. A. Piotrowski, S. Tulaczyk, N. K. Larsen, and C. Brædstrup, A new methodology to simulate subglacial deformation of water-saturated granular material, Cryosphere 9, 2183 (2015).

[41] C.-T. Hsu and P. Cheng, Thermal dispersion in a porous medium, Int. J. Heat Mass Transf. 33, 1587 (1990).

[42] P. L. Bhatnagar, E. P. Gross, and M. Krook, A model for collision processes in gases. I. Small amplitude processes in charged and neutral one-component systems, Phys. Rev. 94, 511 (1954).

[43] J. Zhang, L. Wang, and J. Ouyang, Lattice Boltzmann model for the volume-averaged Navier-Stokes equations, Europhys. Lett. 107, 20001 (2014).

[44] Z. Guo, C. Zheng, and B. Shi, Discrete lattice effects on the forcing term in the lattice Boltzmann method, Phys. Rev. E 65 , 046308 (2002).

[45] A. Vikhansky and I. Ginzburg, Taylor dispersion in heterogeneous porous media: Extended method of moments, theory, and modeling with two-relaxation-times lattice Boltzmann scheme, Phys. Fluids 26, 022104 (2014).

[46] I. Ginzburg, D. d'Humières, and A. Kuzmin, Optimal stability of advection-diffusion lattice Boltzmann models with two re- 
laxation times for positive/negative equilibrium, J. Stat. Phys. 139, 1090 (2010).

[47] Z. Guo and C. Shu, Lattice Boltzmann Method and Its Applications in Engineering, Vol. 3 (World Scientific, Singapore, 2013).

[48] S. Succi, The Lattice Boltzmann Equation: For Fluid Dynamics and Beyond (Oxford University Press, Oxford, UK, 2001).

[49] T. Krüger, H. Kusumaatmaja, A. Kuzmin, O. Shardt, G. Silva, and E. M. Viggen, The Lattice Boltzmann Method (Springer, Berlin, 2017).

[50] M.-L. Maier, T. Henn, G. Thaeter, H. Nirschl, and M. J. Krause, Multiscale simulation with a two-way coupled lattice
Boltzmann method and discrete element method, Chem. Eng. Technol. 40, 1591 (2017).

[51] T. Baldock, M. Tomkins, P. Nielsen, and M. Hughes, Settling velocity of sediments at high concentrations, Coastal Eng. 51, 91 (2004).

[52] S. H. Kim and H. Pitsch, A generalized periodic boundary condition for lattice Boltzmann method simulation of a pressure driven flow in a periodic geometry, Phys. Fluids 19, 108101 (2007).

[53] Z. Guo and C. Shu, Lattice Boltzmann Method and Its Applications in Engineering, Advances in Computational Fluid Dynamics, Vol. 3 (World Scientific, Singapore, 2013). 